\title{
Mixed acoustic-entropy combustion instabilities in gas turbines
}

\author{
Emmanuel Motheau ${ }^{1} \dagger$, Franck Nicoud ${ }^{2}$ \\ and Thierry Poinsot ${ }^{3}$ \\ ${ }^{1}$ CERFACS, 42 av. Gaspard Coriolis, 31057 Toulouse, France \\ ${ }^{2}$ CNRS I3M, University Montpellier II, 34095 Montpellier, France \\ ${ }^{3}$ CNRS - Institut de mécanique des fluides, 1 Allée du Professeur Camille Soula, 31000 \\ Toulouse, France
}

(Received ?; revised ?; accepted ?. - To be entered by editorial office)

A combustion instability in a combustor terminated by a nozzle is analysed and modelled based on a low order Helmholtz solver. A Large Eddy Simulation (LES) of the corresponding turbulent, compressible and reacting flow is first performed and analysed based on Dynamic Mode Decomposition (DMD). The mode with the highest amplitude shares the same frequency of oscillation as the experiment (approx. $320 \mathrm{~Hz}$ ) and shows the presence of large entropy spots generated within the combustion chamber and convected down to the exit nozzle. The lowest purely acoustic mode being in the range $700-750 \mathrm{~Hz}$, it is postulated that the instability observed around $320 \mathrm{~Hz}$ stems from a mixed entropy/acoustic mode where the acoustic generation associated with entropy spots being convected throughout the choked nozzle plays a key role. The DMD analysis allows to extract from the LES results a low-order model that confirms that the mechanism of the low-frequency combustion instability indeed involves both acoustic and convected entropy waves. The Delayed Entropy Coupled Boundary Condition (Motheau et al. 2014) is implemented into a numerical Helmholtz solver where the baseline flow is assumed at rest. When fed with appropriate transfer functions to model the entropy generation and convection from the flame to the exit, the Helmholtz/DECBC solver predicts the presence of an unstable mode around $320 \mathrm{~Hz}$, in agreement with both LES and experiments.

\section{Introduction}

It has been long known that combustion instabilities can lead to high amplitude oscillations of all physical quantities (pressure, velocity, temperature, etc.). A classical mechanism for combustion instability is a constructive coupling between acoustic waves and the unsteady combustion that arises when pressure and heat release fluctuations are in phase (see Rayleigh 1878; Lieuwen \& Yang 2005; Culick \& Kuentzmann 2006). Another mechanism that may also support self-sustained instabilities relies on the acoustic perturbations induced by entropy spots (temperature and/or mixture heterogeneities say) being generated in the flame region and evacuated through the downstream nozzle. This latter mechanism generates indirect combustion noise which may be perceptible outside of the combustion chamber (Marble \& Candel 1977; Cumpsty \& Marble 1977; Bake et al. 2009; Howe 2010; Miles 2010; Leyko et al. 2011; Duran et al. 2013). However, the contribution of this mechanism to the unstable behaviour of air-flames remains controversial.

$\dagger$ Email address for correspondence: emmanuel.motheau@adelaide.edu.au 
- First, purely thermo-acoustic arguments usually allow to explain the observed instabilities very nicely in most cases (McManus et al. 1993; Candel et al. 2009).

- Second, basic experiments studied in labs are usually operated at atmospheric pressure (Lawn et al. 2004; Kornilov et al. 2009; Karimi et al. 2009; Kim et al. 2010) to ease the technological issues. This means that the combustor chamber is not connected to a choked nozzle, eliminating any contribution of the indirect noise effect ab initio.

Knowledge about mixed acoustic-entropy combustion instabilities goes back to the history of rocket engines and ramjets in the 1950s. Earlier works focused on the downstream nozzle that ends the flame tube. Tsien (1952) formulated a transfer function at the entrance of the nozzle via the derivation of an impedance, modelling the damping or increasing contribution of the device. The problem was reformulated later by Zinn (1972) and Morfey (1973) in terms of acoustic energy balance. Multiple works dedicated to the characterisation of the nozzle impedance emerged in the 70's. Bohn (1977) for the subsonic case, as well as Marble \& Candel (1977) for both subsonic and supersonic cases, derived the reflection/transmission coefficients for impinging acoustic and entropic waves in the zero frequency limit (compact nozzle assumption). This approach was later extended to the non-compact cases by Moase et al. (2007) and Goh \& Morgans (2011), and generalised for both subsonic and choked flows with and without shock wave by Duran \& Moreau (2013).

It must be noted that the influence of the nozzle on the combustion zone located upstream is most of the times not accounted for. For example, experiments (Bell et al. 1973; Zinn et al. 1973) or numerical studies (Bloy 1979) focused on the acoustic response of nozzles for several flow parameters (influence of the Mach number or amplitudes of temperature spot) and its sensitivity to the geometry details (sharpness, area contraction effects). Purely mathematical models (Ffowcs-Williams \& Howe 1975) addressed the problem from the noise radiation point of view via the derivation of Green functions. Concerning the interaction mechanism between the combustion and the indirect noise generated at nozzles, to the authors knowledge the first attempt to develop an analytical theory goes back to the works of Abouseif et al. (1984), closely followed by Keller et al. (1985). They found that the time lag characteristic of temperature spots detached from the flame and convected to the nozzle could add a new family of eigenmodes affecting the overall stability of the system. However, as recalled by $\mathrm{Yu}$ et al. (1991), there was a lack of experimental evidence on the contribution of a mixed, convective mode to the flame instability. Despite the fact that this latter work focused on the interaction between vortex and nozzle, the results can be extrapolated to the interaction between entropy and acoustic modes and constitute a first step to outline that the instability mechanism relies on the combination of acoustic and convective time lags. Contrary to the indirect combustion noise, the vortex shedding and its interaction with the acoustics is an important part of the stability of solid rocket engines and many studies are devoted to this subject (Vuillot 1995; Chedevergne et al. 2012). An experimental study that enlightened the role of the indirect noise on combustion instability was performed by Macquisten \& Dowling (1994), who observed that the flame burning in a simple tube could be strongly affected by the addition of a nozzle. However it was just an observation which was not followed by a modelling effort.

The works cited above were motivated by the study of rocket engines and ramjets. The subject has taken a renewed gain of interest with the development of lean premixed combustor for gas turbines, especially in the aeronautical application where these engines cover a wide range of operating points and are more prone to combustion instabilities due to the technological choices. The complexity of such systems has naturally led to the development of low-order methods to predict thermo-acoustic instabilities. The most 
comprehensive works about low-order modelling of combustion instability (Keller 1995; Dowling \& Stow 2003; You et al. 2005) rely on the resolution of a wave equation governing the oscillatory field in the chamber. Complemented by experiments (Hield et al. 2009; Yu et al. 2010; Sisco et al. 2011) these low order models show that a new family of instability modes that depends on the mean flow velocity may exist. However, as pointed out by Eckstein et al. (2006), one important issue is that the influence of indirect combustion noise is not systematic and a low-frequency instability may appear even without the presence of a nozzle, and vice-versa. Some elements of response were provided by Polifke et al. (2001) who showed that entropy and acoustic waves may have a constructive or destructive phase dependency, as well as Sattelmayer (2003) who argued that entropy spots are submitted to strong dilution (mixing) due to the highly turbulent nature of the flow in practical combustors. Recent numerical study by Morgans et al. (2013) suggests however that the amount of dissipation of entropy spots convected by a turbulent flow may remain moderate. The importance of the entropy wave dissipation in mixed modes introduced a new mechanism to consider in models. It did not have to be considered for purely acoustic unstable modes which are only marginally affected by wave dissipation. The effects of dissipation and dispersion on unstable mixed modes have been investigated in a recent study by Goh \& Morgans (2013). They confirmed that entropy waves can destabilise a stable combustor, but can also have a stabilising effect on an unstable configuration. Moreover, some configurations can exhibit a mode switch between unstable modes. However, despite the fundamental interest of these studies, they still rely on 1D low-order methods and simple academic configurations.

Only a few numerical studies have been performed to reproduce a mixed entropyacoustic combustion instability in an air-breathing combustor. Zhu et al. (2001) and Yao et al. (2012) considered an academic combustor and applied the Reynolds Averaged Navier-Stokes (RANS) methods although the accuracy of this approach when dealing with reacting turbulent flows is highly questionable. Unsteady RANS computations have been performed by Hochgreb et al. (2013) in an academic combustor. These authors found that the mechanism of convection of entropy spots to the downstream nozzle has a keyrole on the establishment of a self-sustained thermoacoustic oscillation, whose computed frequency match the experiments. Large-Eddy Simulation (LES) is now recognised as appropriate (although computationally demanding) to deal with flame dynamics related issues, with or without coupling with acoustics (see Pitsch (2006); Schmitt et al. (2007); Wolf et al. (2012); Gicquel et al. (2012), among many others). LES was used to investigate the noise generated by the impact of acoustic and entropy waves on nozzles or turbine stages (see Bodony 2009; Duran et al. 2013). However to the authors knowledge no LES study deals specifically with a mixed entropy-acoustic instability.

LES computations are very computationally demanding and faster tools are required in the design process of new burners. A natural approach is to characterise the stable/unstable modes in the frequency domain. An approximate linear wave equation for the amplitude $\hat{p}(\boldsymbol{x})$ of the pressure perturbations $p^{\prime}(\boldsymbol{x}, t)=\hat{p}(\boldsymbol{x}) \exp (-j \omega t)$ in reacting flows may be derived from the Navier-Stokes equations (see Poinsot \& Veynante 2011) and reads:

$$
\bar{\rho}(\boldsymbol{x}) \bar{c}^{2}(\boldsymbol{x}) \nabla \cdot\left(\frac{1}{\bar{\rho}(\boldsymbol{x})} \nabla \hat{p}(\boldsymbol{x})\right)+\omega^{2} \hat{p}(\boldsymbol{x})=j \omega(\gamma(\boldsymbol{x})-1) \hat{q}(\boldsymbol{x})
$$

where $\hat{q}(\boldsymbol{x})$ is the amplitude of the unsteady heat release $q^{\prime}(\boldsymbol{x}, t)=\hat{q}(\boldsymbol{x}) \exp (-j \omega t), \gamma$ the heat capacity ratio, $\bar{\rho}$ is the density of the baseline flow, $\bar{c}$ the speed of sound and $\omega$ is the complex angular frequency. In order to close the problem, the flame is modelled as a purely acoustic element based on a $n-\tau$ type of model (Crocco 1952); Eq. (1.1) 
is referred to as the inhomogeneous Helmholtz equation and corresponds to a non-linear eigenvalue problem which can be solved by using appropriate discretisation techniques and numerical algorithms (Nicoud et al. 2007).

Eq. (1.1) relies on the so-called zero-Mach-number assumption stating that the mean velocity is very small compared to the speed of sound. Nicoud \& Wieczorek (2009) suggest that the domain of validity of the zero mean flow assumption might be rather small. One reason for this is that Eq. (1.1) does not support entropy waves. Thus the acoustic generation due to the entropy spot being accelerated in the nozzle/turbine located downstream of the combustion chamber is not accounted for and mixed modes cannot be captured. Recall that the production of sound by acceleration of entropy fluctuations is a key phenomenon when dealing with combustion noise; neglecting this acoustic source when studying thermoacoustic instabilities is thus questionable.

Nevertheless, this somewhat restrictive assumption is necessary to derive a wave equation for the thermoacoustic perturbations superimposed to a non isentropic baseline flow, the alternative being to use the complete set of Linearised Euler Equations (LEE) (see Nicoud \& Wieczorek 2009). Unfortunately, this would make the computational effort needed to compute the thermoacoustic modes significantly larger (five coupled equations being solved for) than what is required when dealing with Eq. (1.1). More importantly, the LEEs couple the different modes of fluctuations that may interact and produce a combustion instability. Consequently solving the LEEs would not be as informative as solving the Helmholtz equation with or without additional terms related to the mean flow. Being able to (partly) account for the non-zero Mach number effects without relying on the LEEs is therefore highly desirable to discriminate if an instability is purely of acoustic nature or if it relies on a mixed acoustic/entropy mode of oscillation.

The objectives of this paper are the following:

- build and analyse a large-eddy simulation data base to establish the presence of mixed modes in a realistic combustion chamber;

- develop and validate a methodology to capture the mean flow effects within the zero-Mach-number framework (Eq. (1.1));

- apply the obtained low-order method to the same 3D industrial-like combustor.

First, the industrial configuration, the numerical set-up and the associated low frequency instability are presented in Sec. 2. A purely acoustic study is performed with the help of a zero-Mach-number Helmholtz solver, demonstrating that a classical thermoacoustic approach cannot represent the observed low-frequency mode. In Sec. 3 a Dynamic Mode Decomposition (DMD) is applied to the LES results in order to investigate the presence of an entropy-acoustic coupling via the reconstruction of a reduced-order model. A proper formalism is then introduced in Sec. 4 in order to account for the entropy-acoustic coupling within the zero Mach number framework by the derivation of a Delayed Entropy Coupled Boundary Condition (DECBC). Finally, results from the zero Mach number Helmholtz solver with the DECBC approach are presented to illustrate the potential of the method.

\section{Combustor analysis}

\subsection{Description of the experimental configuration}

The case considered in this study is a combustor developed by the SAFRAN Group for aero-engine applications. Figure 1 shows the combustion chamber and the casing with primary and dilution holes although some parts (shaded) of the geometry are not displayed for confidentiality reasons (these details do not affect the main features pre- 

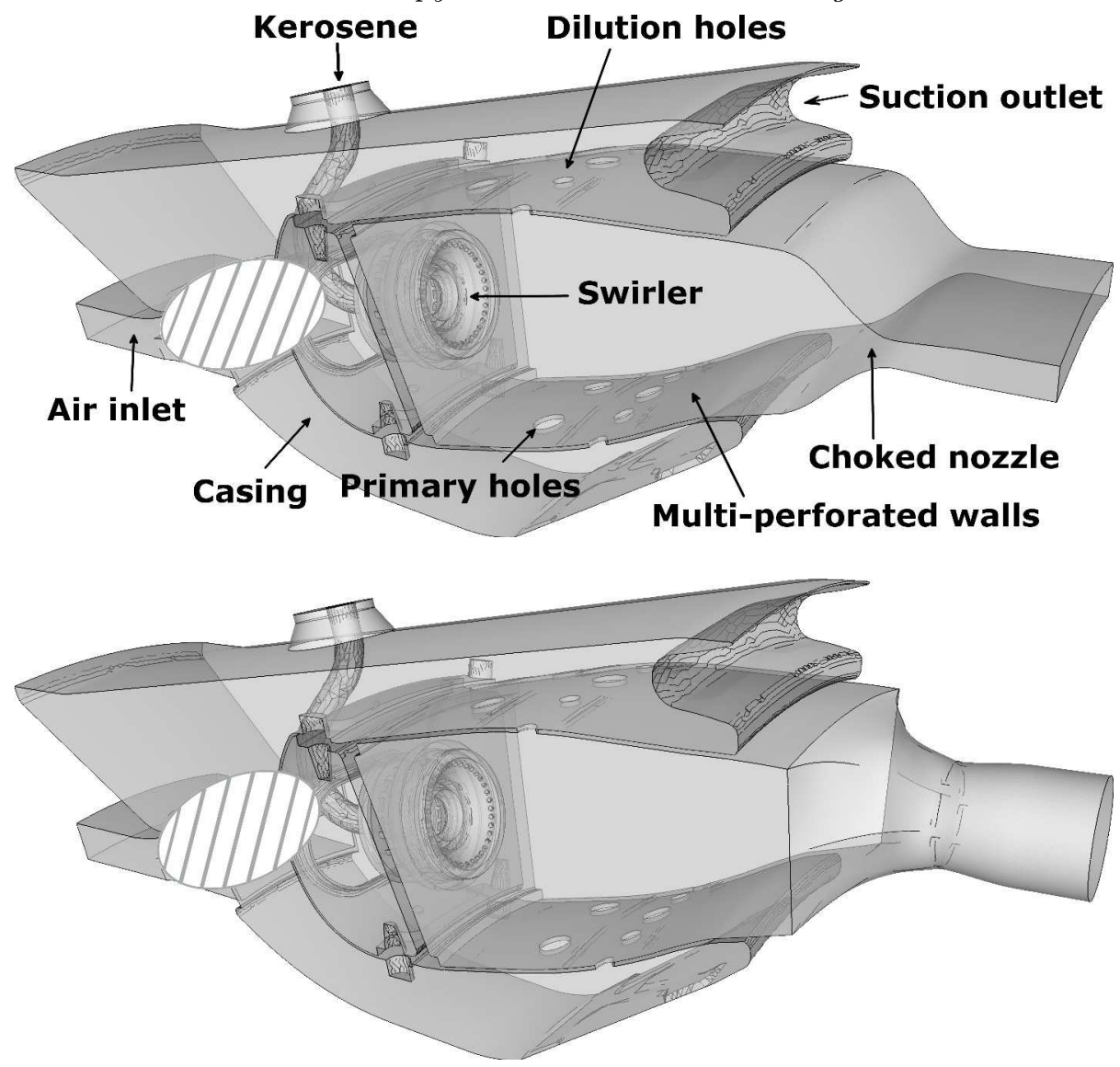

FIgURE 1. Description of the configuration of interest. One sector of the azimuthal SAFRAN combustor is represented. The high pressure distributor downstream of the combustion chamber is modelled by a nozzle with equivalent cross section and which is either rectangular (top) or circular (bottom).

sented and conclusions reached). The air inlet connected to the upstream compressor is also displayed. Note that the downstream high pressure distributor which connects the combustor to the turbine in the actual experiment is not represented here nor directly accounted for in the remaining of the paper. Instead it is modelled by a choked nozzle with equivalent cross section area. For choked conditions, this ensures that the chamber sees an outlet condition close to the actual one. The whole mass-flow rate is not entering into the nozzle: approx. $16 \%$ is routed downstream of the surrounding casing (modelled in the simulation by suction walls). The fuel line is also visible, as well as the swirled injector used to mix fuel and air and to generate the recirculation zone which stabilises the flame. In the engine, several injectors are mounted all around the azimuthal combustion chamber although only one sector with one injector is displayed in the figure. Note that this geometry is representative of a typical aero-engine gas turbine combustor, as studied in other works (see Dowling \& Stow 2003).

Experiments were performed on the present configuration to study the effect of liquid water ingestion. Depending on the ratio between the mass flow rates of water and air, the overall stability of the engine evolves, the combustor becoming increasingly unstable as the mass flow rate of water increases. As the temperature of the ingested liquid water 
is approx. $300 \mathrm{~K}$, it is postulated that all liquid water is vaporised when flowing through the compressor. Adding water to the incoming air has two effects: (1) a decrease of the air temperature and (2) a dilution effect due to the presence of vaporised water in the inlet air. Both effects decrease the flame speed and can affect the instability mechanism but effect (2) is small compared to a decrease of the inlet temperature of the combustion air (the flame speed changes like $T^{2}$ ). Although not detailed in the present paper, several LES computations have been performed for different inlet boundary conditions:

- one which takes into account the mass fraction of vaporised water in the gas entering into the combustor,

- one where only pure air enters the combustion chamber.

These tests show that the main effect is the decrease of the inlet temperature and that the injection of vaporised water has a limited effect: no significant differences were observed on the flame dynamics or the frequency and amplitude of the instability. Therefore we considered only the first one by using a reaction mechanism where no chemical effect of water is included and only the air temperature plays a role. In consequence, the effect of liquid water is only taken into account in the present study by decreasing the air entering into the combustor. It is possible to link the inlet temperature $T_{\text {inlet }}$ to the mass flow rates of liquid water and air by a simple enthalpy balance. The engine is found stable for $T_{\text {inlet }}$ approx. $760 \mathrm{~K}$ but unstable for $T_{\text {inlet }}$ approx. $560 \mathrm{~K}$. In unstable cases the measured pressure signal exhibits strong fluctuations at approximately $330 \mathrm{~Hz}$ and with a maximum amplitude of approx. $5 \times 10^{4} \mathrm{~Pa}$.

\subsection{Numerical set-up}

To analyse this instability, large eddy simulations (LES) were performed using the AVBP solver (CERFACS 2009). The latter is based on a cell-vertex formulation and a set of finite element / finite volume schemes for unstructured meshes. A two-step TaylorGalerkin scheme ( $3^{\text {nd }}$ order in time and space) was retained (Colin \& Rudgyard 2000). Two regimes were computed by LES, corresponding to two representative operating conditions investigated experimentally at SAFRAN: one which contains a strong instability at nearly $330 \mathrm{~Hz}\left(T_{\text {inlet }}=557 \mathrm{~K}\right.$; operating point $\left.C o l d\right)$ and one which shows no instability $\left(T_{\text {inlet }}=762 \mathrm{~K}\right.$; operating point $\left.H o t\right)$. In each case, only one angular sector (out of 20) was computed, axiperiodic conditions being prescribed at the lateral boundary. It is consistent with the experimental data showing that the low frequency instability is not an azimuthal mode. Turbulent combustion was represented with the Dynamic Thickened Flame Model developed by Colin et al. (2000). A simple two-step kinetic scheme was used to represent kerosene-air chemistry (Franzelli et al. 2010). The global power is approx. $1.4 \times 10^{6} \mathrm{~W}$ while the global equivalence ratio is approx. 0.3 , which is characteristics of lean combustors. The mass flow rate of air entering into the domain is $\dot{m}_{\mathrm{O}}=1.37 \mathrm{~kg} / \mathrm{s}$, while the Reynolds number in the inlet channel is approx. $7.5 \times 10^{5}$. The mass flow rate of Kerosene is $\dot{m}_{\mathrm{F}}=0.032 \mathrm{~kg} / \mathrm{s}$.

Boundary conditions are imposed through the NSCBC formulation (Poinsot \& Lele 1992) to control spurious acoustic reflections. The solid walls are adiabatic and a classical logarithmic law is imposed to mimic the near-wall shear stress (Schmitt et al. 2007). The coincident walls between the combustion chamber and the casing are actually multiperforated and modelled in the simulation by an adiabatic homogeneous suction/injection boundary condition (Mendez \& Nicoud 2008a,b). Only the hydrodynamics effects of the multiperforated walls are taken into account by the model, not the associated acoustic damping. For each LES computations data are recorded upon a period of $120 \mathrm{~ms}$ after the whole system reaches a limit cycle state. As the expected instability phenomenon has 


\begin{tabular}{|c|c|c|c|c|c|c|}
\hline Run & Operating Point & Baseline mesh & Fine mesh & Circular Nozzle & Flat Nozzle & Regime \\
\hline 1 & Cold & $x$ & & $x$ & & Unstable \\
\hline 2 & Hot & $x$ & & $x$ & & Stable \\
\hline 3 & Cold & $\times$ & & & $\times$ & Unstable \\
\hline 4 & Hot & $x$ & & & $\times$ & Stable \\
\hline 5 & Cold & & $x$ & $x$ & & Unstable \\
\hline 6 & Hot & & $x$ & $x$ & & Stable \\
\hline
\end{tabular}

TABLE 1. Summary of the LES computations performed on the two operating points Cold $\left(T_{\text {inlet }}=557 \mathrm{~K}\right)$ and $\operatorname{Hot}\left(T_{\text {inlet }}=762 \mathrm{~K}\right)$ depending on the nozzle geometry (circular or flat) and the mesh (baseline mesh approx. 5.6 millions cells, fine mesh approx. 15 millions cells). The last column denotes if the computed flow was found stable or unstable. Recall that the experiment is unstable for Cold and stable for Hot.

a frequency of approx. $330 \mathrm{~Hz}$, this latter choice ensures that the averaging procedure of physical fields and signal processing like Fourier transforms cover at least 40 cycles.

As mentioned in Sec. 2.1, the turbine is replaced by a choked nozzle with equivalent cross section area. The natural question that arises is whether the response of the nozzle to entropic (temperature) perturbations is sensitive to the three-dimensional nature of the temperature hot-spots. Two nozzle geometries were tested so as to ensure the robustness of the results to the shape of the nozzle: a flat one and a circular one, depicted in the top and bottom of Fig. 1, respectively.

The fundamental characteristic of the LES methodology is that the mesh grid acts as a spatial filter for the turbulence spectrum, the larger scales being solved numerically on the discretised domain while the effect of the smallest scales is modelled by a subgrid scale turbulent viscosity $\mu_{t}$. It is then crucial to estimate the sensitivity of the results to the mesh refinement as well as models for $\mu_{t}$. The present study focuses on the instability generated inside the chamber, downstream of the flame. Thus only the pressure amplitude and the frequency of the phenomena are considered as crucial parameters to assess the quality of the LES. No further investigations were performed to quantify the influence of the mesh level refinement as well as the subgrid scale models on the physics of the flow and the flame. Hence, two meshes were tested for the geometry coupled with a circular nozzle: a baseline one (approx. 5.6 millions cells) and a fine one (approx. 15 millions cells). Note that in both meshes the veins inside the swirler as well as the dilution holes are refined to capture the flow in small geometrical details.

Moreover, two subgrid scale models for the turbulent viscosity $\mu_{t}$ have been tested:

- the classical Smagorinsky model (Smagorinsky 1963)

$$
\mu_{t}=\left(C_{S} \Delta\right)^{2} \sqrt{2 \widetilde{S}_{i j} \widetilde{S}_{i j}}
$$

where $\Delta$ denotes the filter characteristic length and $C_{S}=1.8$ is a constant. The turbulent viscosity $\mu_{t}$ is then proportional to the resolved filtered strain tensor $\widetilde{S}_{i j}$. The Smagorinsky model was developed in the 1960's and is extensively tested over a wide range of flow configurations. This model is easy to implement and presents good properties for flows away from solid walls at a low computational cost. Furthermore, it supplies the 
right amount of kinetic energy dissipation in homogeneous isotropic turbulent flows. The Smagorinsky model is also known as being too dissipative in wall bounded flows and to overpredicts the wall-shear stress near solid boundaries. This problem can be avoided by the use of wall-law boundary conditions.

- the Sigma model (Nicoud et al. 2011)

$$
\mu_{t}=\left(C_{\sigma} \Delta\right)^{2} \frac{\sigma_{3}\left(\sigma_{1}-\sigma_{2}\right)\left(\sigma_{2}-\sigma_{3}\right)}{\sigma_{1}^{2}}
$$

where $C_{\sigma}=1.5$ is a model constant, $\sigma_{1} \geqslant \sigma_{2} \geqslant \sigma_{3} \geqslant 0$ are the singular values of the resolved velocity gradient tensor. The $\sigma$ model was developed to overcome some drawbacks observed on most of the static models without using additional test filter. Indeed, it presents the interesting properties to vanish in various laminar flow configurations (pure shear, pure rotation, pure dilatation/contraction, ...) for which no subgrid scale activity is expected. It also has the property to vanish with the proper asymptotic behaviour near solid boundaries.

Table 1 presents a summary of the LES computations performed on the two operating points depending on the nozzle geometry and the mesh refinement. In order to discriminate either the effect of the nozzle or the inlet temperature, all numerical and physical parameters in the computations are strictly the same.

\subsection{LES results}

Figure 2 displays typical snapshots (instantaneous temperature fields) of the LES for the operating points Cold and Hot. Contours of the heat release rate $\left(1 \times 10^{9} \mathrm{~W} . \mathrm{m}^{-3}\right)$ are superimposed to the temperature field. Both cases exhibit a typical swirled flame with a central recirculation zone around the axis of the swirler. An interesting feature of the flame is that chemical reactions occur also near the cooling film, as well as along the dilution jet where fresh air flows into the combustor. Theoretically, the role of the dilution holes is to confine the flame in the upstream zone by suddenly reducing the fuel/air ratio. In both cases, this objective is reached and the temperature drops down after the dilution jets. However, in the case of the operating point Cold (Fig. 2 top), a hot pocket of temperature is released after the dilution jets and the mixture is still reacting when convected to the downstream nozzle by the mean flow. A set of temperature snapshots is displayed in Fig. 3 showing how this hot pocket is created, convected and ejected by the nozzle. On the contrary in the case of the operating point Hot, the dilution jets play their role and the flame is confined (Fig. 2 bottom). Only burned gas are convected to the downstream nozzle.

The pressure signal is recorded at a probe (denoted A, see Fig. 2) located at the entrance of the nozzle. Results for the operating points Cold and Hot are depicted in Fig. 4 by the solid and dotted line, respectively. In the case of operating point Cold, the pressure exhibits strong harmonic fluctuations at a fundamental low-frequency close to the experimental value $(330 \mathrm{~Hz})$. The mean pressure is approx. $1.65 \times 10^{6} \mathrm{~Pa}$. The amplitude of the limit cycle is quite large and is in the range of pressure fluctuations amplitudes measured in the experiments (approx. $5 \times 10^{4} \mathrm{~Pa}$ ). In the case of operating point Hot, the pressure signal is typical of broadband noise with a smaller fluctuation level. The mean pressure is also higher (approx. $1.76 \times 10^{6} \mathrm{~Pa}$ ) than for the Cold case.

In order to assess the influence of the shapes of the downstream nozzle, two computations have been performed using the baseline mesh for the operating point Cold, both with the flat and circular geometry (Runs \#1 and \#3, see Table 1). Figure 5 presents the amplitude of the Fourier transform of each signal recorded at probe A. The mean pressure value has been subtracted from processed signals. The first two peaks at $320 \mathrm{~Hz}$ 

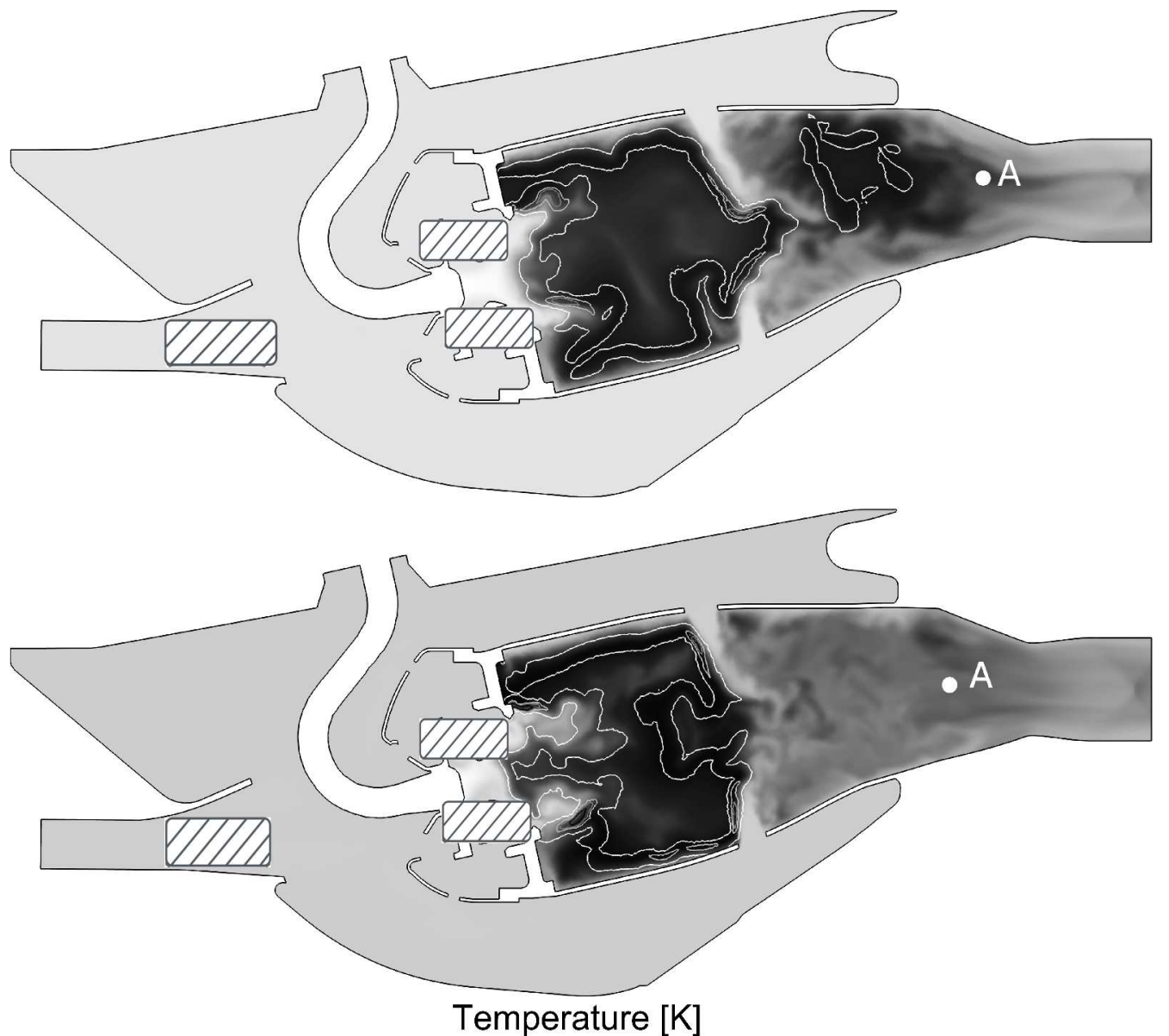

Temperature $[\mathrm{K}]$

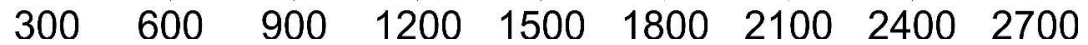

FIGURE 2. Instantaneous fields of temperature with superimposition of one contour of Heat Release rate (white line, $1 \times 10^{9} \mathrm{~W} \cdot \mathrm{m}^{-3}$ ). Top: operating point Cold. Bottom: operating point Hot. The snapshots are from Runs \#5 and \#6, respectively (see table 1).

and $640 \mathrm{~Hz}$ are virtually the same for both nozzle geometries while the frequency of the third and fourth peaks (approx. $960 \mathrm{~Hz}$ and approx. $1280 \mathrm{~Hz}$, respectively) are slightly shifted. This shows that the estimation of the instability is not sensitive to the nozzle geometry for the low-frequency mode of interest. Only the circular nozzle geometry will be considered in the remaining of the paper.

As explained above in Sec. 2.2, operating points Cold and Hot were solved with the two levels of mesh refinement (Runs \#1, \#2, \#5 and \#6 in Table 1). Figure 6 presents the modulus of the Fourier transform of the pressure signal recorded at probe A for Runs \#5 (fine mesh) and \#1 (baseline mesh). The mean pressure value has been subtracted from processed signals. Results for the first unstable mode are similar even if the amplitude of fluctuations computed with the baseline mesh is slightly under-predicted and their frequency slightly shifted. This can be explained by the fact that the baseline mesh is more dissipative and that entropy waves are dissipated too rapidly during their convection to the downstream nozzle. The modulus of the Fourier transform of the pressure signal 

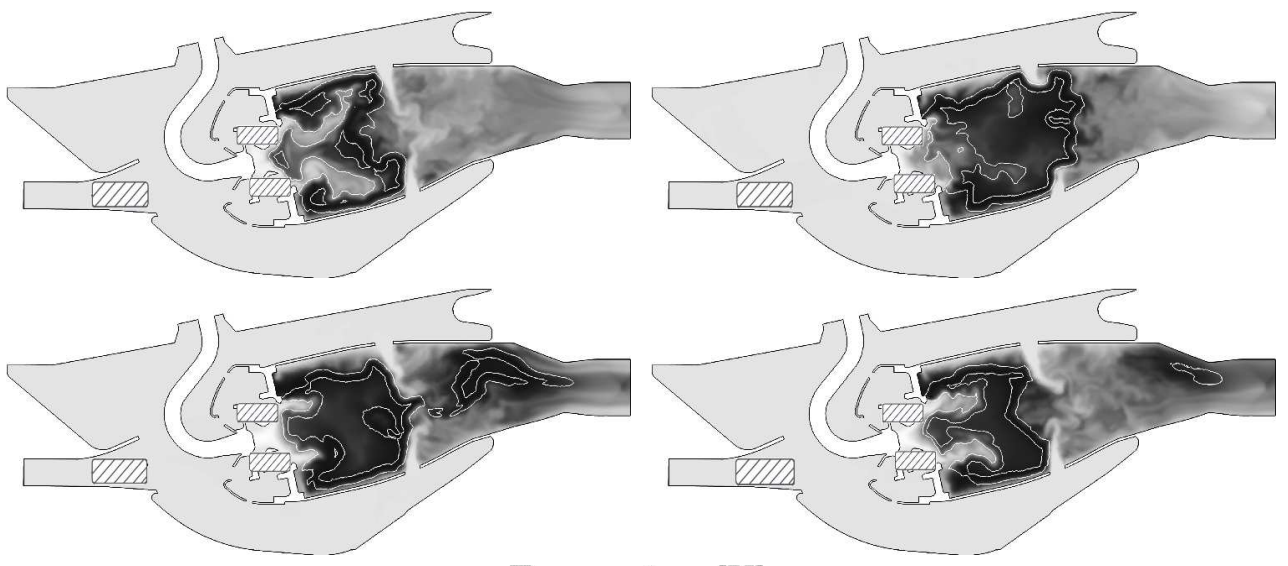

Temperature $[\mathrm{K}]$

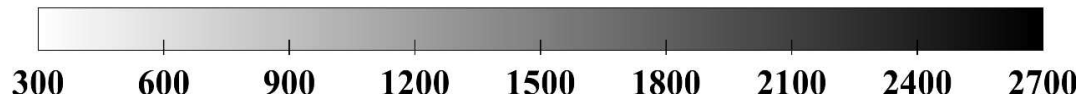

FIGURE 3. Instantaneous fields of temperature with superimposition of one contour of Heat Release rate (white line, $1 \times 10^{9} \mathrm{~W} \cdot \mathrm{m}^{-3}$ ). Full cycle of the evolution of the temperature. Top left: the flame is confined in the primary zone. Top right: a hot pocket of temperature is created under the dilution holes. Bottom left: the hot pocket is convected downstream. Bottom right: the hot pocket is ejected through the exit nozzle.

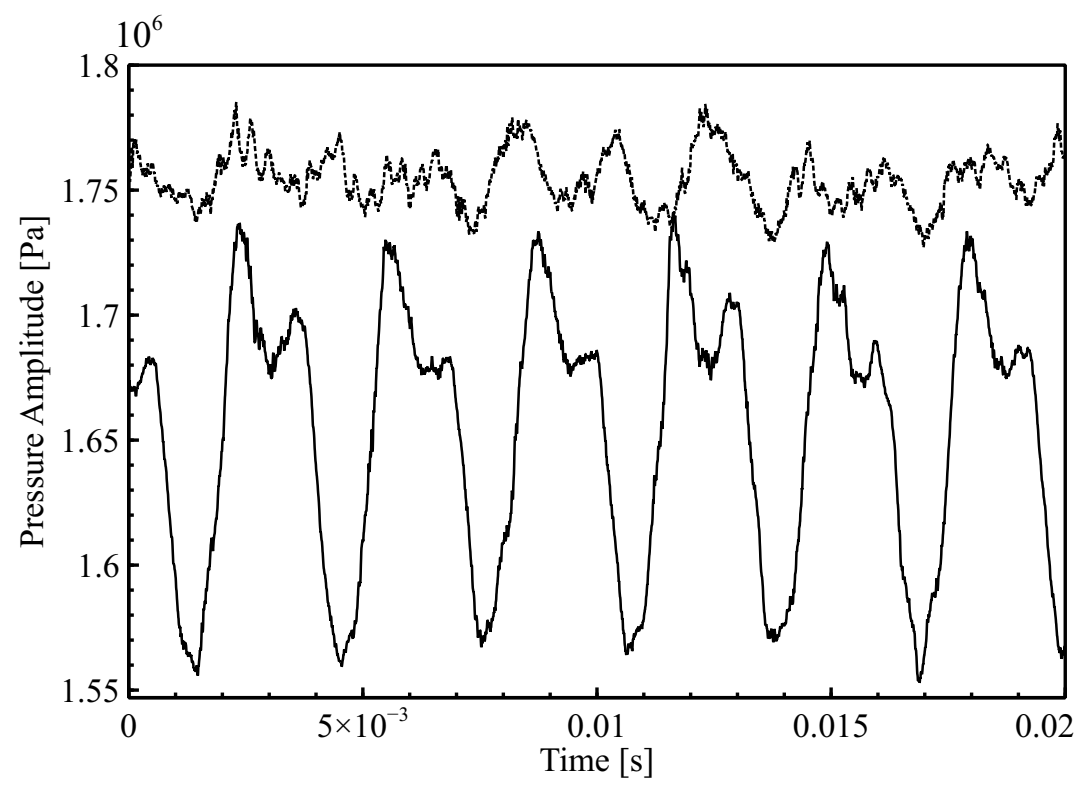

Figure 4. Pressure signals from LES data at probe A and for the circular nozzle geometry. Solid line ( - ): Operating point Cold, Run \#5. Dotted line ( …....): Operating point Hot, Run \#6. The mean pressure is approx. $1.65 \times 10^{6} \mathrm{~Pa}$ and $5 \times 10^{4} \mathrm{~Pa}$ for operating points Cold and Hot, respectively.

recorded at probe A for the operating point Hot is also displayed in Fig. 6. A peak is present at the frequency of approx. $435 \mathrm{~Hz}$ and has an amplitude of $2500 \mathrm{~Pa}$. It is remarkable that pressure fluctuations for the operating point Hot are very weak compared to operating point Cold, confirming the capacity of LES to capture and discriminate 


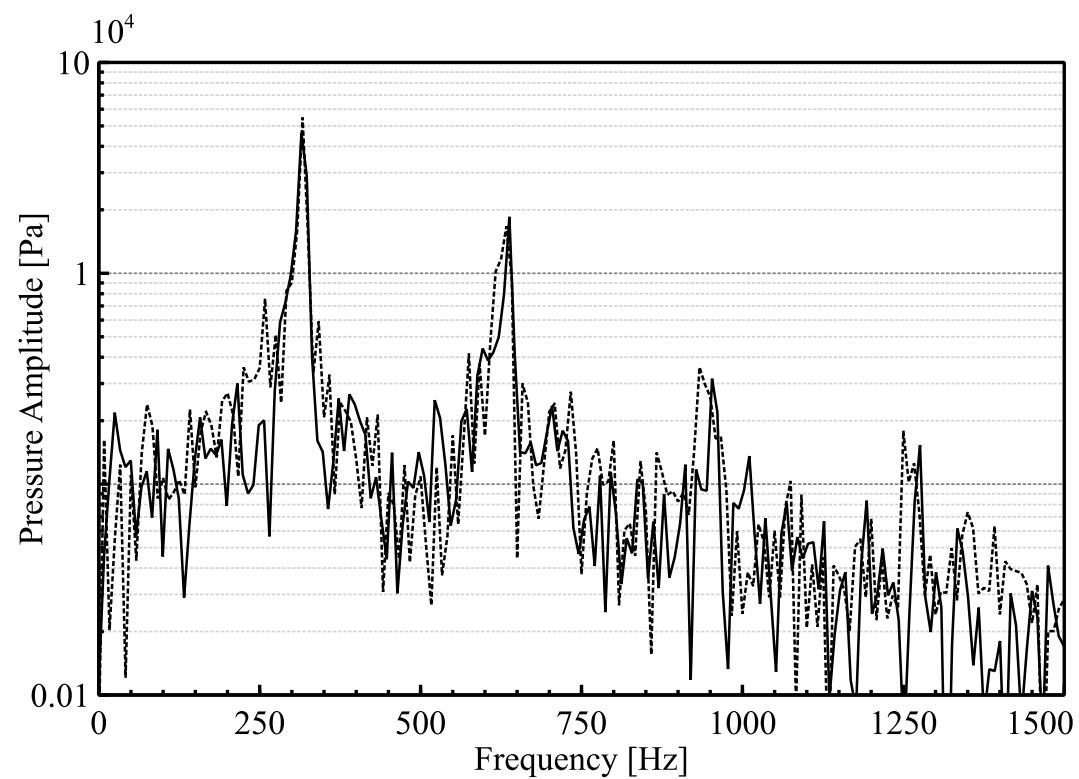

FiguRE 5. Spectra of pressure signals from LES data at probe A performed on the coarse mesh and for the operating point Cold. The mean pressure value has been subtracted from processed signals. Solid line ( - ): circular nozzle, Run \#1 (see top of Fig. 1 and table 1). Dotted line ( ): flat nozzle, Run \#3 (see bottom of Fig. 1 and table 1).

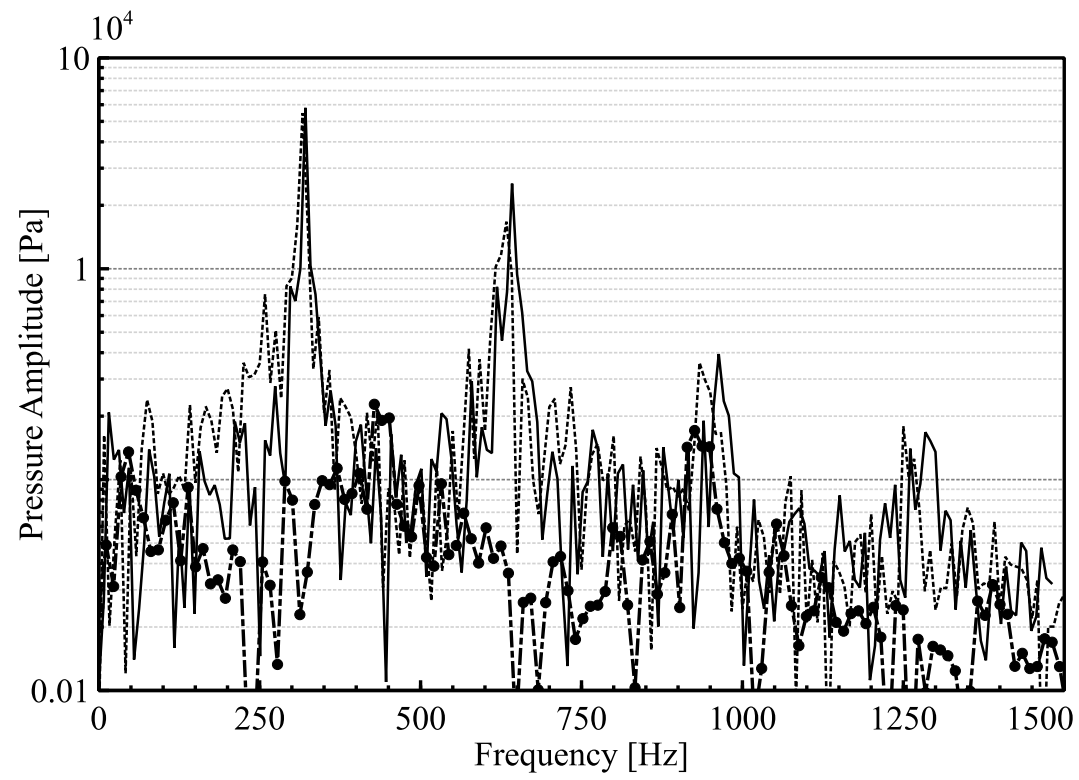

FIGURE 6. Spectra of pressure signals from LES data at probe A and for the circular nozzle geometry. The mean pressure value has been subtracted from processed signals. Solid line ( - ): Cold and fine mesh (Run \#5). Dotted line ( .......): Operating point Cold and coarse mesh (Run \#1). Dot-dashed line with black circles $(-\bullet-)$ : Operating point Hot and fine mesh (Run \#6). 


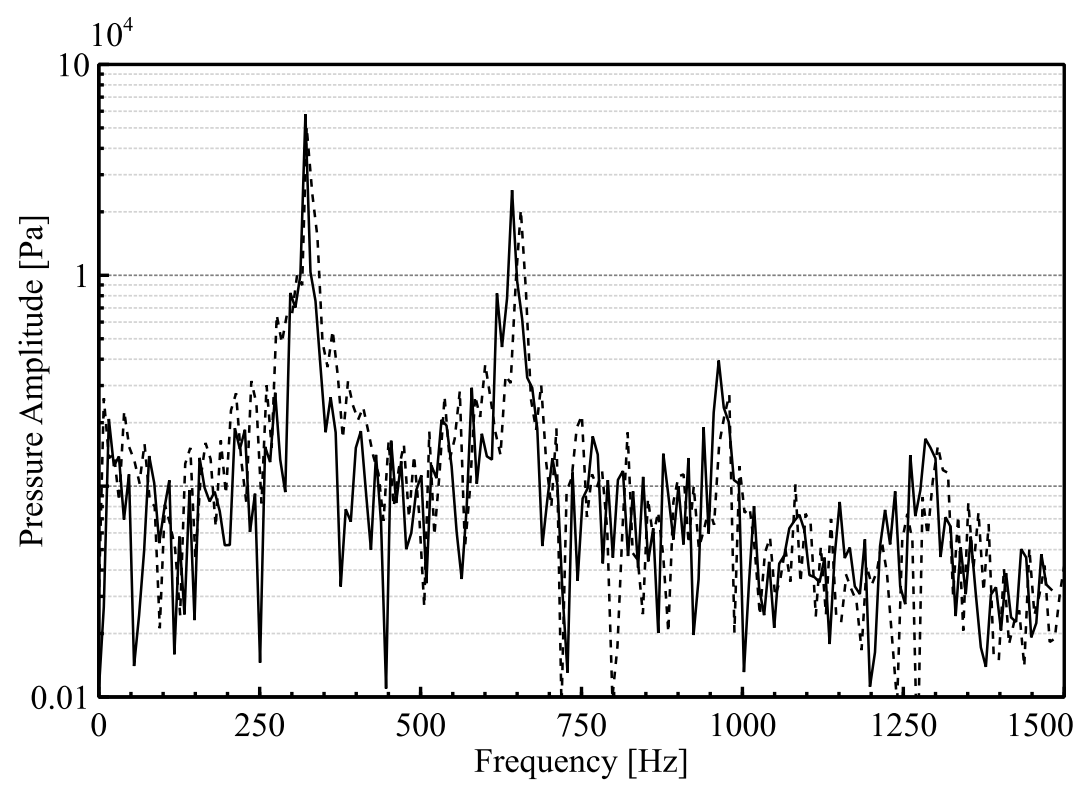

FIGURE 7. Spectra of pressure signals from LES data at probe A and for the circular nozzle geometry and fine mesh (Operating point Cold, Run \#5). The mean pressure value has been subtracted from processed signals. Solid line ( - ): Smagorinsky model (Smagorinsky 1963). Dashed line ( - - ): Sigma model (Nicoud et al. 2011).

between stable and unstable regimes on both meshes of Table 1. Although not depicted in Fig. 6, results for Run \#2 are similar to Run \#6.

Additionally, to make sure that the instability mechanism predicted by LES is independent of the subgrid scale model for the turbulent viscosity $\mu_{t}$, the Run \#5 was computed with the classical Smagorinsky and the Sigma models as described at Sec. 2.2. Results for the modulus of the Fourier transform of the pressure signal recorded at probe A are depicted in Fig. 7. The mean pressure value has been subtracted from processed signals. Results for the first unstable mode are similar both in frequency and amplitude. For the second unstable mode, results with the Sigma model slightly differ: the frequency is shifted of a few Hertz while the amplitude is under-estimated. However for the phenomena of our interest at approx. $320 \mathrm{~Hz}$, Fig. 7 demonstrates that the results are independent of the modelling of the turbulent viscosity $\mu_{t}$. Note that a run without subgrid scale model has been attempted on both baseline and fine meshes. These computations are unstable and lead to a rapid numerical divergence: this demonstrates that the numerical viscosity is weak and that subgrid scale models for the turbulent viscosity $\mu_{t}$ are essential. Finally, as results are very similar even when the mesh is refined and the subgrid scale model is changed, the LES computations are considered robust to reproduce the instability phenomena accurately.

\subsection{Helmholtz analysis}

An acoustic analysis is performed by using the AVSP software (Nicoud et al. (2007)) that solves numerically the Helmholtz equation (1.1) as an eigenvalue problem. The unsteady heat release rate $\hat{q}(\boldsymbol{x})$ constitutes a forcing source related to the acoustic/flame coupling. It is modelled based on a $n-\tau$ type of model (Crocco (1952)):

$$
\hat{q}(\boldsymbol{x})=n_{\text {local }}(\boldsymbol{x}) \hat{\mathbf{u}}\left(\boldsymbol{x}_{r e f}\right) e^{j \omega \tau_{\text {local }}(\boldsymbol{x})} \cdot \boldsymbol{n}_{\text {ref }}
$$


Eq. (2.3) essentially relates the unsteady heat release $\hat{q}$ to the acoustic velocity $\hat{\mathbf{u}}$ along the direction of the unity vector $\boldsymbol{n}_{r e f}$ at reference location $\boldsymbol{x}_{r e f}$. The space dependent amplification factor (unit [J.m $\left.{ }^{-4}\right]$ ) and a time delay (unit $[\mathrm{s}]$ ) are $n_{\text {local }}$ and $\tau_{\text {local }}$ respectively. The $n_{\text {local }}-\tau_{\text {local }}$ parameters can be estimated experimentally or by analytical models. However in the present study, these parameters are directly extracted from the LES database by post-processing the heat release rate and velocity signals obtained for the self sustained case Cold. Note that the Helmholtz equation only involves the acoustic pressure $\hat{p}$ and that the velocity vector $\hat{\mathbf{u}}$ can be deduced from $\hat{p}$ by using the momentum equation under the zero-Mach-number assumption: $j \omega \bar{\rho} \hat{\mathbf{u}}=\nabla \hat{p}$. Finally Eq. (1.1) solved by the Helmholtz solver is rewritten as follows:

$\bar{\rho}(\boldsymbol{x}) \bar{c}^{2}(\boldsymbol{x}) \nabla \cdot\left(\frac{1}{\bar{\rho}(\boldsymbol{x})} \nabla \hat{p}(\boldsymbol{x})\right)+\omega^{2} \hat{p}(\boldsymbol{x})=(\gamma(\boldsymbol{x})-1) n_{\text {local }}(\boldsymbol{x}) e^{j \omega \tau_{\text {local }}(\boldsymbol{x})} \frac{\nabla \hat{p}\left(\boldsymbol{x}_{r e f}\right) \cdot \boldsymbol{n}_{r e f}}{\bar{\rho}\left(\boldsymbol{x}_{r e f}\right)}$

The sound speed $\bar{c}$, the mass density $\bar{\rho}$ and the heat capacity ratio $\gamma$ are local variables that are estimated from time averaged LES solutions.

Solutions of Eq. (2.4) are basically a set of eigenmodes which are characterised by a complex frequency and associated shapes of pressure distribution. The imaginary part of the complex frequency is interpreted as the stability rate of the eigenmode. Care must be taken concerning the interpretation of the stability rate. Usually the temporal evolution of a combustion instability is decomposed in two phases (Poinsot \& Veynante 2011): (1) an exponential linear growth followed by (2) a non-linear limit cycle. The stability rate computed by the Helmholtz equation only provides information about the linear transient phase. Validating this piece of information against LES data would require measuring the growth rate from temporal signals during the transient linear phase which is very challenging when dealing with a turbulent flow. A common way of checking the validity of a low-order thermoacoustic analysis is to assume that any mode with a (strong) positive growth rate should lead to a limit cycle with similar frequency and spatial structure. At the same time, any linear mode with negative or marginally positive growth rate is expected to be damped without forming a limit cycle.

Note that although the Helmholtz analysis is conducted in the linear domain, the models are fed with parameters (the field of speed of sound, n-tau parameters for the active flame or DECBC) computed from the non-linear state (the limit cycle). This is of course a limitation of the present approach but it is usual practice made in thermoacoustic studies (Martin et al. 2006; Wolf et al. 2012). A posteriori results tend to show that the linear study is able to capture unstable/stable modes with a coherent estimation of the frequency. Recent studies (Selimefendigil \& Polifke 2011; Silva et al. 2013) demonstrate that it is possible to capture the non-linear behaviour when combining several transfer functions computed from the linear regime.

\subsubsection{Acoustic boundary conditions}

Since Eq. (2.4) assumes no mean flow, it is necessary to restrict the study of thermoacoustic instabilities to only the combustion chamber (where the Mach number is always small). As depicted in Fig. 8, the domain is truncated at the outlet, before the nozzle, where the Mach number is $M \approx 0.1$. It is then crucial to take into account the proper acoustic environment of the combustor, as for example the presence of a compressor or a turbine; this is illustrated in Fig. 9 where $Z_{\mathbf{u} p}^{0}$ stands for the proper acoustic impedances that must be imposed at the edges of the Helmholtz $(M=0)$ domain in order to account for the acoustic waves transmission/reflection due to the compressor and turbine. Note 


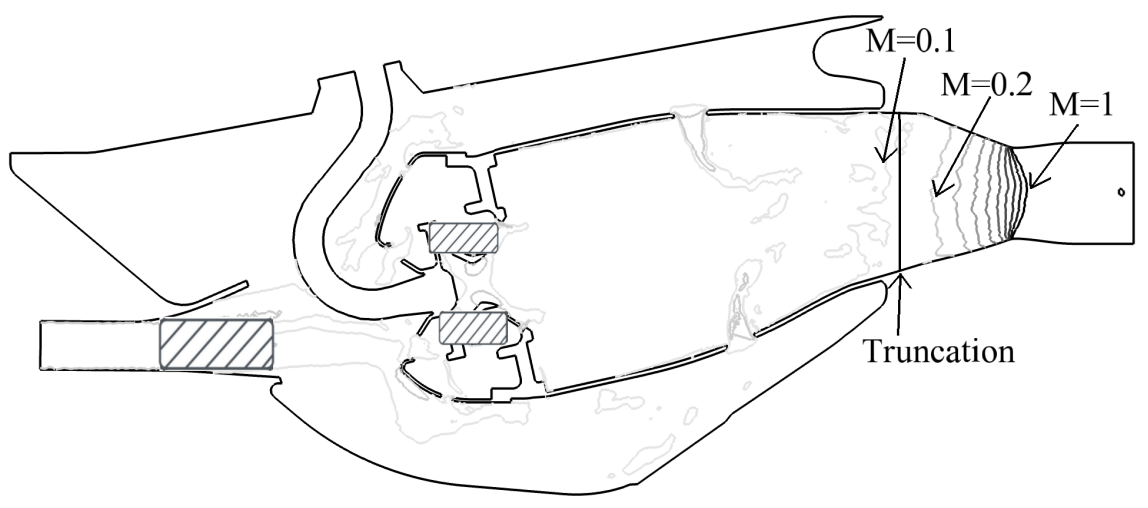

FiguRE 8. Isocontour of Mach number in the combustor for the computation at operating point Cold. The black line represents the location where the domain is truncated $(M \approx 0.1)$.

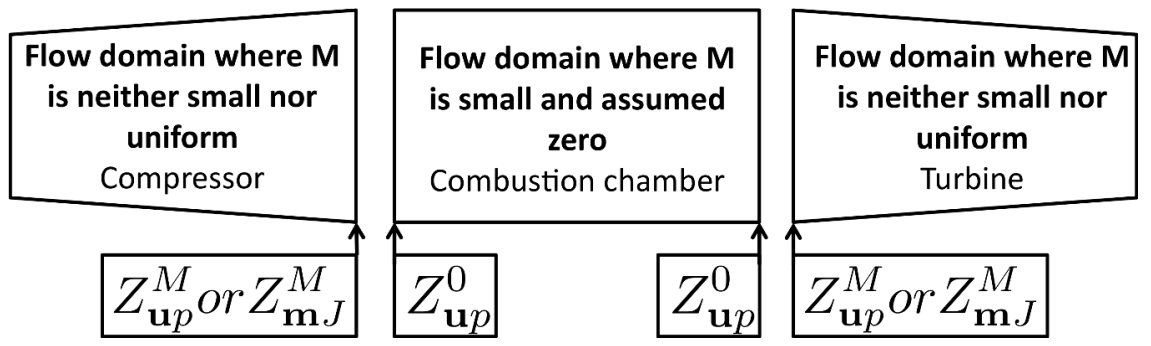

FIGURE 9. Schematic view of the modelling strategy: Instead of solving for the LEEs over the whole domain, the Helmholtz equation is solved over the combustion chamber only, the acoustic environment from compressor and turbine being accounted for by imposing relative impedances.

that the Mach number in the surrounding elements is not necessary small, especially in the turbine where the flow is highly accelerated. The natural question that arises is then: what is the proper way to impose an impedance that models nonzero-Mach-number $(M \neq 0)$ elements to a Helmholtz solver based on the zero-Mach-number assumption? This subject has been handled by the authors in previous works (see Motheau et al. 2012, 2014) and is briefly recalled in the following.

When dealing with acoustics in the presence of mean flow effects, the impedance over a boundary of normal $\mathbf{n}$ may be formulated in two different ways:

$$
\overline{\rho c} Z_{\mathbf{u} p}^{M} \hat{\mathbf{u}} \cdot \mathbf{n}-\hat{p}=0 \quad \text { or alternatively } \quad \frac{\bar{c}}{\bar{\rho}} Z_{\mathbf{m} J}^{M} \hat{\mathbf{m}} \cdot \mathbf{n}-\hat{J}=0
$$

where $\hat{\mathbf{m}}=\bar{\rho} \hat{\mathbf{u}}+\overline{\mathbf{u}} \hat{p} / \bar{c}^{2}$ and $\hat{J}=\hat{p} / \bar{\rho}+\overline{\mathbf{u}} \cdot \hat{\mathbf{u}}$ are the (complex amplitudes of the) mass flow rate and total enthalpy respectively, and $Z_{\mathbf{u} p}^{M}$ and $Z_{\mathbf{m} J}^{M}$ are the impedances associated with variables $(\hat{\mathbf{u}}, \hat{p})$ and $(\hat{\mathbf{m}}, \hat{J})$ respectively. The choice of this latter set of fluctuating state variables is commonly made in the aeroacoustic community (see Peters et al. 1993; Karlsson \& Åbom 2010) because they are themselves independent on the flow when placed in a wave equation, contrary to the pressure or the velocity which are locally dependent 
on the flow velocity (see Doak 1998). The superscript $M$ in Eq (2.5) denotes the fact that the impedances are relevant to acoustic elements where the mean flow is not at rest (as in a compressor or turbine, see Fig. 9). Of course, the impedances $Z_{\mathbf{u} p}^{M}$ and $Z_{\mathbf{m} J}^{M}$ are two different complex valued numbers although they represent the same physical element (compressor or turbine). For example, a perfect tube end where the acoustic pressure $\hat{p}$ is zero would correspond to $Z_{\mathbf{u} p}^{M}=0$ but $Z_{\mathbf{m} J}^{M}=\mathbf{M} \cdot \mathbf{n}=\overline{\mathbf{u}} \cdot \mathbf{n} / \bar{c}$. More generally, these impedances are related by the following relation:

$$
Z_{\mathbf{m} J}^{M}=\frac{Z_{\mathbf{u} p}^{M}+\mathbf{M} \cdot \mathbf{n}}{1+Z_{\mathbf{u} p}^{M} \mathbf{M} \cdot \mathbf{n}}
$$

Now, since the Helmholtz equation is solved for in the combustion chamber where the mean flow is assumed at rest, a boundary impedance $Z_{\mathbf{u} p}^{0}$ should be imposed in order to account for the effects of the compressor/turbine on the acoustics. Even if $\hat{p}$ is the primary variable (see Eq. (1.1)) in the combustion chamber, the proper impedance $Z_{\mathbf{u} p}^{0}$ to be imposed at the edge of the chamber is not necessarily $Z_{\mathbf{u} p}^{M}$ : even if the Mach number is zero in the chamber, it is not in the nozzle and its effect should be taken into account when computing $Z_{\mathbf{u} p}^{M}$. A careful analysis of the energy flux through the interface between the combustion chamber and the outer acoustic elements (Motheau et al. 2014) shows that a proper choice is $Z_{\mathbf{u} p}^{0}=Z_{\mathbf{m} J}^{M}$ because this introduces an error of order $M^{2}$ on the energy flux, while $Z_{\mathbf{u} p}^{0}=Z_{\mathbf{u} p}^{M}$ introduces an error of order $M$. For example, if a choked feeding line is located upstream of the combustion chamber, the mass flux is constant $\left(\hat{\mathbf{m}} \cdot \mathbf{n}=0, Z_{\mathbf{m} J}^{M}=\infty\right)$ and the proper boundary condition for the Helmholtz domain is simply $Z_{\mathbf{u} p}^{0}=\infty$. Similarly, if the feeding line imposes the velocity instead of the mass flux $\left(\hat{\mathbf{u}} \cdot \mathbf{n}=0, Z_{\mathbf{m} J}^{M}=1 / \mathbf{M} \cdot \mathbf{n}, Z_{\mathbf{u} p}^{M}=\infty\right)$, the proper boundary impedance is $Z_{\mathbf{u} p}^{0}=1 / \mathbf{M} \cdot \mathbf{n}$ and not $\infty$

In the present study, the nozzle impedance $Z_{\mathbf{u} p}^{M}$ was computed based on the analytical approach of Duran \& Moreau (2013) which solves the quasi one-dimensional LEE via the Magnus expansion. The main advantage of this latter method over the Marble \& Candel (1977) compact approximation is that it gives for any given complex frequency the exact amplitude of the impedance and the related phase relations, while keeping a negligible computational cost. The impedance $Z_{\mathbf{u} p}^{M}$ was then corrected for Mach effects using Eq. 2.6 to provides $Z_{\mathbf{m} J}^{M}$.

\subsubsection{Results}

Several computations of the operating points Cold and Hot have been performed with the AVSP Helmholtz solver (Nicoud et al. 2007). In all the cases, a baseline mesh (approx. $10^{5}$ nodes) was generated from the LES grid in order to represent the geometry properly. Additionally, a zero normal acoustic velocity condition was imposed over all boundaries except a) over the chamber exit where the nozzle impedance $Z_{\mathbf{m} J}^{M}$ (see Sec. 2.4.1) is imposed, and $\mathbf{b}$ ) over the air inlet which is modeled by a constant mass flow rate $\hat{\mathbf{m}} \cdot \mathbf{n}=0$, i.e. $Z_{\mathbf{m} J}^{M}=\infty$.

The sound speed $\bar{c}$ is computed from the averaged temperature field given by LES and interpolated on the coarse acoustic mesh. In the cold region such as the casing where fresh air is flowing, the sound speed is approx. $300 \mathrm{~m} . \mathrm{s}^{-1}$ and it reaches approx. $900 \mathrm{~m} . \mathrm{s}^{-1}$ in hot regions. The fields of $n_{\text {local }}$ and $\tau_{\text {local }}$ are computed by taking the Fourier transform of the signals of fluctuating heat release rate and velocity, so as to form $\hat{q}(\boldsymbol{x})$ at each point of the domain as well as $\hat{\mathbf{u}}\left(\boldsymbol{x}_{r e f}\right) \cdot \boldsymbol{n}_{\text {ref }}$ at a reference point $\boldsymbol{x}_{r e f}$ located inside the swirler. For Helmholtz computations of the case Cold, Fig. 10 presents the fields of $n_{\text {local }}$ and $\tau_{\text {local }}$ extracted at frequency $320 \mathrm{~Hz}$ of the thermoacoustic instability. The reference 

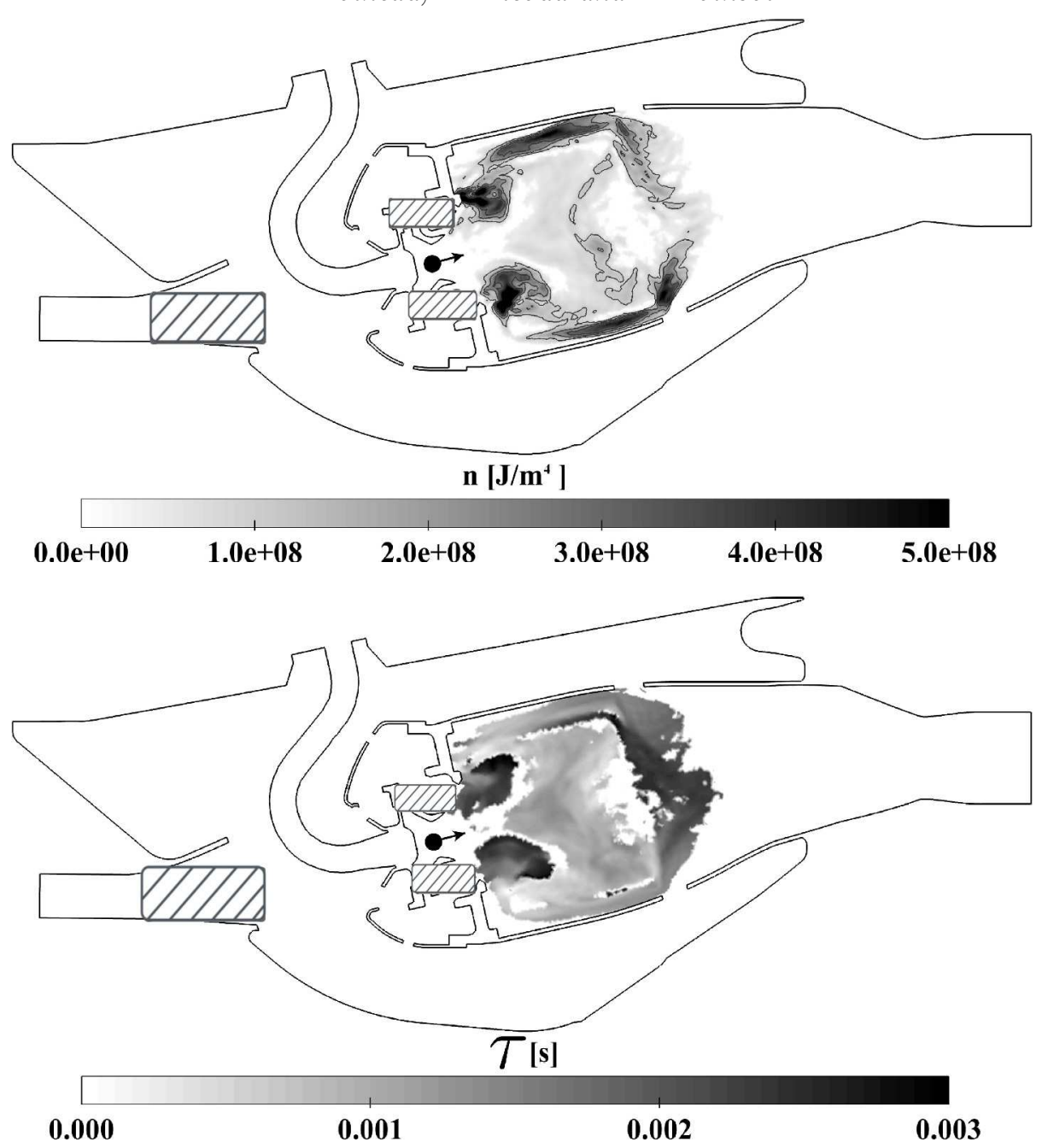

FIGURE 10. Fields of $n_{\text {local }}$ and $\tau_{\text {local }}$ computed at $320 \mathrm{~Hz}$ and for the operating point Cold, Run \#5. The black dot indicates the location of the reference point $\boldsymbol{x}_{r e f}$ for the acoustic velocity.

point $\boldsymbol{x}_{r e f}$ for the velocity as well as the normal vector of reference $\boldsymbol{n}_{r e f}$ are also shown. For computations of the case Hot, the fields of $n_{\text {local }}$ and $\tau_{\text {local }}$ are extracted at $440 \mathrm{~Hz}$ (not shown), which is the frequency in the LES signals (see Fig. 6) where most of the activity occurs.

The main properties of the computations performed as well as the results for the first mode are gathered in Table 2 and Table 3 for operating points Cold and Hot, respectively. The Active Flame column reports whether or not the flame-acoustic coupling (the RHS term of Eq. (2.4)) is included. The Boundary Conditions column reports whether the value of $Z_{\mathbf{u} p}^{0}$ (see section 2.4.1) at the outlet is set to $\infty$ (simple $\hat{\mathbf{m}} \cdot \mathbf{n}=0$ condition) or computed from the analytical nozzle theory of Duran \& Moreau (2013). Computations of the operating points Cold and Hot feature the same behaviour. The first eigenmode obtained for operating point Cold is in the range of $690-730 \mathrm{~Hz}$, very far from the instability at $320 \mathrm{~Hz}$ encountered in the experiments and the LES computations. Similarly, the first mode computed for operating point Hot is found in the range of $817-840 \mathrm{~Hz}$, 


\begin{tabular}{c|c|c|c|c} 
Name & Outlet Impedance $Z_{\mathbf{m} J}^{M}$ & Active Flame & Frequency $[\mathrm{Hz}]$ & Growth/Decay rate $\left[\mathrm{s}^{-1}\right]$ \\
\hline Run A & $\infty$ & no & 731 & 0 \\
Run B & $\infty$ & yes & 703 & -18 \\
Run C & from Duran \& Moreau (2013) & no & 721 & -27 \\
Run D & from Duran \& Moreau (2013) & yes & 690 & -42
\end{tabular}

TABLE 2. Results for Helmholtz computations of operating point Cold $\left(T_{\text {inlet }}=557 \mathrm{~K}\right)$.

\begin{tabular}{c|c|c|c|c} 
Name & Outlet Impedance $Z_{\mathbf{m} J}^{M}$ & Active Flame & Frequency [Hz] & Growth/Decay rate $\left[\mathrm{s}^{-1}\right]$ \\
\hline Run A & $\infty$ & no & 840 & 0 \\
Run B & $\infty$ & yes & 830 & 0 \\
Run C & from Duran \& Moreau (2013) & no & 826 & -30 \\
Run D & from Duran \& Moreau (2013) & yes & 817 & -28
\end{tabular}

TABLE 3. Results for Helmholtz computations of operating point $H o t\left(T_{\text {inlet }}=762 \mathrm{~K}\right)$.

contrary to the (limited) thermoacoustic activity observed at approx. $435 \mathrm{~Hz}$. Runs B show that the coupling with the flame only shifts the frequency by a few Hertz and has a damping effect on the acoustic modes. Runs $\mathrm{C}$ show that the use of more accurate boundary conditions has a limited effect on the frequency of the first eigenmode. Using an active flame model and proper boundary conditions within a Helmholtz solver framework is the most employed method to calculate thermoacoustic modes and to draw the stability map of a reactive system. However Runs D confirm that such a method totally fails to capture the desired unstable mode of interest in this particular study. This result suggests that the instability encountered in the present combustor is not of purely acoustic nature and supports the idea that it relies on a convective path. The next natural question is: how to evidence this path if it exists and how to model it in the purely zero-Mach number acoustic framework? The next section is devoted to an advanced post-processing of the LES by using Dynamic Mode Decomposition.

\section{Dynamic Mode Decomposition}

\subsection{Theoretical background}

Experiments or simulations of flows often lead to complex, dynamic and non-linear systems which can not be analysed directly by simple visualisation or statistical techniques. Advanced methods have emerged during the last decades to provide a detailed analysis of many flow fields. In this context, the extraction of coherent structures from the flow as well as their stability analysis have become essential. The time-evolution of a flow is 
described by:

$$
\frac{\mathrm{d} \mathbf{v}(t)}{\mathrm{d} t}=A \mathbf{v}(t)
$$

where $\mathbf{v}$ is a vector containing the physical variables representative of the flow and $A$ a linear operator. When integrated between $t$ and $t+\Delta t$ while keeping $A$ constant, Eq. (3.1) leads to:

$$
\mathbf{v}(t+\Delta t) \approx e^{\Delta t A} \mathbf{v}(t)
$$

In practice, the flow is time-discretised and represented by a set of $N$ vectors whose $M$ components $(M>>N)$ contain any relevant quantity taken either from experimental or numerical data at $N$ different instants. Mathematically speaking, the amplitude of all the variables at all points are represented by the following sequence of state vectors:

$$
V_{1}^{N}=\left\{\mathbf{v}_{1}, \mathbf{v}_{2}, \mathbf{v}_{3}, \cdots, \mathbf{v}_{N}\right\}
$$

where the subscript and superscript denotes the time index of the first and last snapshot, respectively. If the state vectors contain enough information, the flow dynamics can be approximated by a linear operator $\widetilde{A}=e^{\Delta t A}$ such that :

$$
\mathbf{v}_{n+1}=\widetilde{A} \mathbf{v}_{n} \quad, \quad \forall n
$$

The $\widetilde{A}$ operator may be studied through its eigenvectors $\widetilde{\Phi}_{n}$ and eigenvalues $\widetilde{\lambda}_{n}$ which provide the model structure and frequency/growth rate respectively. In practice, the eigenvalue problem:

$$
\widetilde{A} \widetilde{\Phi}_{n}=\widetilde{\lambda}_{n} \widetilde{\Phi}_{n}
$$

can not be solved since $\widetilde{A}$ is either unknown or too large. The Dynamic Mode Decomposition (DMD) developed by Schmid (2010) is an algorithm to approximate the matrix $\widetilde{A}$. The DMD is an extension of a previous method presented by Rowley et al. (2009) and based on the Arnoldi algorithm. Eqs. (3.3) and (3.4) may be expanded into the following relation:

$$
\widetilde{A} V_{1}^{N-1}=V_{2}^{N}=V_{1}^{N-1} S+r e_{N-1}^{T}
$$

where $r$ is the residual vector which tends to 0 when the number of snapshots $N$ increase, $e_{N-1}$ is the $(N-1)^{t h}$ unit vector and the matrix $S$ is of companion type with:

$$
S=\left(\begin{array}{ccccc}
0 & & & & a_{1} \\
1 & 0 & & & a_{2} \\
& \ddots & \ddots & & \vdots \\
& & 1 & 0 & a_{N-2} \\
& & & 1 & a_{N-1}
\end{array}\right)
$$

where the coefficient $\left\{a_{1}, a_{2}, a_{3}, \cdots, a_{N-1}\right\}$ are the only unknowns. The matrix $S$ shifts the snapshot index 1 to $N-1$ and is a low-dimensional representation of the full system. Consequently $S$ and $\widetilde{A}$ share the same eigenvalues since they are similar from Eq. (3.6) if the residual vector in the RHS is indeed negligible. The advantage of considering $S$ is its reduced size $(N-1 \times N-1)$ compared to the size of $\widetilde{A}(M \times M)$. One drawback of the DMD is that the operator $S$ may be ill-conditioned in practice, especially when dealing with noisy signals. Several formulations of $S$ are available in the literature, as for example by expanding $V_{1}^{N-1}$ via a Singular Value Decomposition (SVD) (Schmid 2010) or a QR-decomposition (Schmid et al. 2011). In the present paper, the variant proposed by Chen et al. (2012) is implemented and described as follows. The operator $S$ 
is reformulated by using Eq. (3.6):

$$
S=\left(V_{1}^{N-1}\right)^{-1} V_{2}^{N}
$$

Introducing first the following SVD: $V_{1}^{N-1}=U \Sigma W^{H}$ where $U$ and $W$ are unitary and $\Sigma$ is diagonal, the Moore-Penrose inversion of $\left(V_{1}^{N-1}\right)^{-1}$ leads to:

$$
\left(V_{1}^{N-1}\right)^{-1}=W \Sigma^{-1} U^{H}
$$

Introducing the substitution $U=V_{1}^{N-1} W \Sigma^{-1}$ into Eq. (3.9) and replacing the whole expression into Eq. (3.8) gives:

$$
S=W \Sigma^{-1} \Sigma^{-1} W^{H}\left(V_{1}^{N-1}\right)^{H} V_{2}^{N}
$$

The operators $W$ and $\Sigma$ may be then simply computed by forming the following diagonalization:

$$
\left(V_{1}^{N-1}\right)^{H} V_{1}^{N-1}=W \Sigma^{2} W^{H}
$$

This construction of $S$ is robust even when $V_{1}^{N-1}$ is nearly rank-deficient, and computing the product $\left(V_{1}^{N-1}\right)^{H} V_{1}^{N-1}$ requires less memory (see Chen et al. 2012) than performing directly a SVD. The last step is to solve the eigenproblem $S s_{n}=\mu_{n} s_{n}$ where $s_{n}$ and $\mu_{n}$ are the eigenvectors and eigenvalues of $S$. From Eq. (3.6) (neglecting the residual term), the relations between the eigenvectors $\widetilde{\Phi}_{n}$ and eigenvalues $\widetilde{\lambda}_{n}$ of $\widetilde{A}$ and their counterparts $s_{n}$ and $\mu_{n}$ are:

$$
\begin{aligned}
& \widetilde{\Phi}_{n}=V_{1}^{N-1} s_{n} \\
& \widetilde{\lambda}_{n}=\mu_{n}
\end{aligned}
$$

Recalling that $\widetilde{A}=e^{\Delta t A}$, the eigenvectors and eigenvalues of $A$ are:

$$
\begin{aligned}
\Phi_{n} & =\widetilde{\Phi}_{n}=V_{1}^{N-1} s_{n} \\
\lambda_{n} & =\frac{\ln \left(\widetilde{\lambda}_{n}\right)}{\Delta t}=\frac{\ln \left(\mu_{n}\right)}{\Delta t}
\end{aligned}
$$

Thus studying the $(N-1) \times(N-1)$ matrix $S$ gives information about the whole flow dynamics $A$.

\subsection{Application of the DMD}

Dynamic Mode Decomposition (DMD) was applied to the LES data in order to better understand the nature of the instability illustrated in Sec. 2.3. For this purpose, 200 snapshots were recorded over a time range corresponding to approx. 8 cycles of the instability, thus leading to a sampling of 28 snapshots by period. This amount of data is sufficient for the DMD to break down the reactive turbulent flow into dynamically relevant structures with periodic evolution over time (Schmid 2010). The input vectors (see Eq. (3.3)) for the DMD algorithm were built from the nodal values of pressure, static temperature, entropy, reaction rate and the three-components of the velocity at each grid point of the mesh used for the LES. Note that the entropy variable, denoted $s$, is not directly an output of the code and has been computed for each node of the domain from LES data with the following expression:

$$
s(\boldsymbol{x})=\frac{C_{p}(\boldsymbol{x})}{\gamma(\boldsymbol{x})} \ln \left(\frac{p(\boldsymbol{x})}{\rho(\boldsymbol{x})^{\gamma(\boldsymbol{x})}}\right)
$$

Although not detailed in the present study, several trials have been performed by 

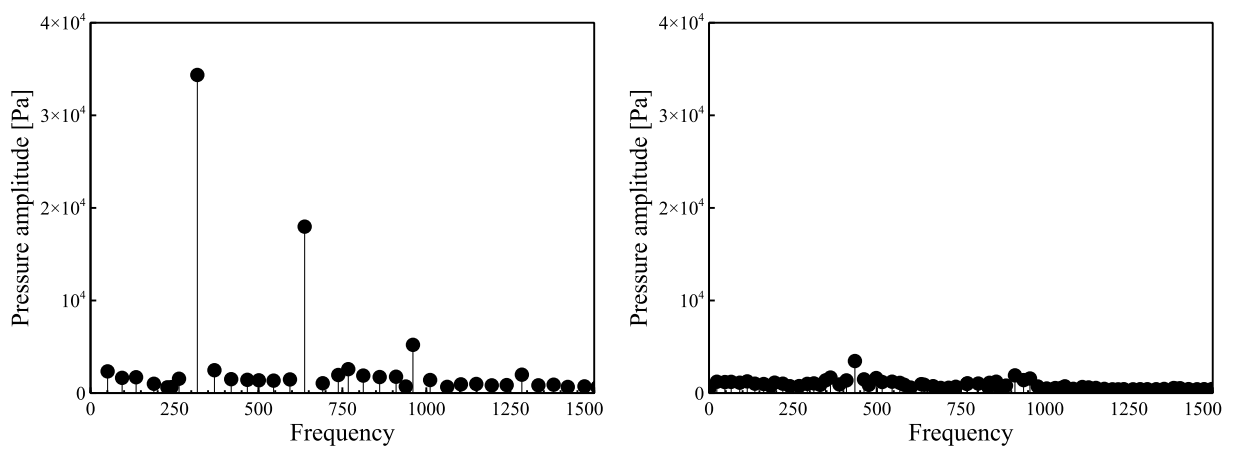

Figure 11. Pressure amplitude $\left(\mathcal{L}^{2}\right.$ norm) spectrum from the DMD analysis of the LES computation. Left: Operating point Cold. Right: Operating point Hot. The frequency is $\Re\left(\lambda_{n}\right)$.

changing the total number of snapshots or by composing the input vector with different sets of variables, showing that the number of variables taken into account in the DMD does not change the conclusions drawn in the remaining of this study. Moreover, variations in the total number of snapshots have a limited impact and only shift the frequency of the most unstable mode by a few Hertz.

The DMD results of the LES for operating points Cold and Hot are depicted in the left and right images of Figs. 11 and 12, respectively. Figure 11 shows the $\mathcal{L}^{2}$ norm $\left\|\Phi_{n}\right\|_{\mathcal{L}^{2}}=\sqrt{\Phi_{n}^{H} \Phi_{n}}$ of the pressure fluctuations associated to the different DMD modes. Figure 12 shows the amplification rate $\Im\left(\lambda_{n}\right)$ of the pressure DMD mode. The DMD analysis for operating point $H o t$ does not exhibit a strong emerging mode. Although not evident, a peak is present at the frequency of $434 \mathrm{~Hz}$ and has an amplitude of approx. $3500 \mathrm{~Pa}$, in agreement with experiments and the Fourier analysis (see Fig. 6). However results for the unstable operating point Cold presented in the left of Fig. 11 exhibit a strong peak at $323 \mathrm{~Hz}$, in good agreement with the Fourier analysis conducted at Sec. 2.3 (see Fig. 6). Additional peaks with a decreasing amplitude are also present at the frequencies $648 \mathrm{~Hz}, 969 \mathrm{~Hz}$ and $1290 \mathrm{~Hz}$. A close look at the spectrum of the amplification rate $\Im\left(\lambda_{n}\right)$ depicted in Fig. 12 shows that each mode featuring a strong pressure activity has a null amplification. This result was expected because the DMD analysis is performed on LES data recorded when the thermoacoustic instability has reached a limit cycle. The transition from the stable to unstable operating points, i.e when the energy is growing inside the combustor, has not been considered in the present study. Note also that the eigenmodes extracted by DMD are related by a simple relation $f_{n}=n f_{0}$, suggesting that the modes of higher frequency are harmonics of a fundamental mode $f_{0}$.

The modulus of the fluctuating temperature field for the DMD mode with the highest amplitude at $323 \mathrm{~Hz}$ is depicted in the top of Fig. 13. The relative phase of the temperature and pressure mode is also recorded along the combustor, from the swirler to the exit nozzle. The path where the data are extracted is represented by a white line and the phase is plotted in the bottom of Fig. 13. Two different zones can be distinguished, each with a specific behaviour of the temperature fluctuations:

- Zone I corresponds to the primary zone between the swirler and the dilution holes. The unsteady swirled flame naturally generates temperature fluctuations, but this latter mechanism is confined in the primary zone $(\mathbf{I})$. Indeed, the flame is surrounded by a zero fluctuating temperature area, suggesting that no pockets of temperature generated by the flame are connected with the zone II downstream of the dilution holes. This statement is supported by the behaviour of the phase, which shifts from $-\pi$ to $\pi$ inside the zone 

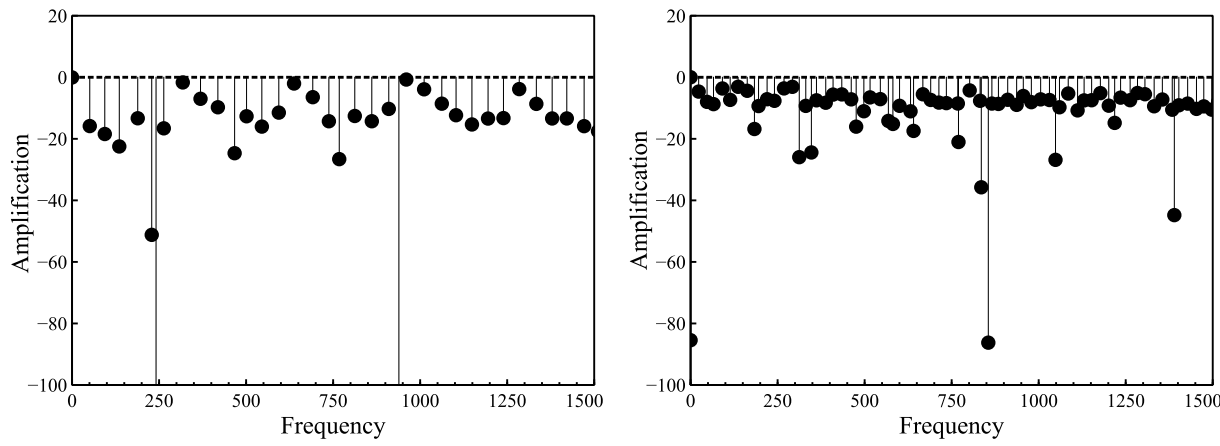

FIGURE 12. Amplification spectrum $\left(\Im\left(\lambda_{n}\right)\right)$ from the DMD analysis of the LES computation. Left: Operating point Cold. Right: Operating point Hot. The frequency is $\Re\left(\lambda_{n}\right)$.

I. Fluctuations of temperature are then rolling up inside the flame and no convection to the downstream zone II occurs.

- Zone II extends from the dilution holes to the exit nozzle. Strong fluctuations of temperature occur along the jets of fresh air that flow from the dilution holes. A large pocket of fluctuating temperature is formed at the top end of the jets, in the middle of the combustor. The phase unrolls monotonously from the formation area of the hot pockets to the downstream nozzle.

The analysis of the modulus and phase of the temperature for the first DMD mode at $323 \mathrm{~Hz}$ suggests that hot pockets of fluctuating temperature are released after the dilution holes and convected to the downstream nozzle. This statement confirms the scenario observed in Fig. 3 showing a series of temperature fields taken from the LES. However, the DMD gives more insight about the origin of these hot pockets: they are not created directly by the flame, which is confined in the primary region, but are generated by the hot gases interacting with the dilution jets.

In order to better understand the mechanism at the origin of the thermoacoustic instability, the fluctuating pressure, temperature and velocity fields from the first DMD mode at $323 \mathrm{~Hz}$ were reconstructed over one period. The four phases displayed in Fig. 14 support the idea that the unstable mode of interest relies, at least partly, on an entropyacoustic coupling. Note that the velocity is represented only by its component along the $y$-axis, i.e. the streaming axis of the dilution jets.

- At phase 0 , the pressure is low everywhere within the combustion chamber and a pocket of cold gas is present downstream of the primary reaction zone, roughly at the middle of the combustion chamber. The velocity perturbations of the dilution jets are maximum: more fresh air is blown into the combustor,

- At phase $\pi / 2$, the cold pocket is convected downstream and the unsteady pressure in the chamber is approximately zero. The velocity perturbations of the jets are very small,

- At phase $\pi$, the cold pocket interacts with the exit nozzle and a new pocket of hot gas is generated downstream of the primary zone, while the fluctuating pressure within the chamber is now positive and the dilutions jets have reached their minimum velocities,

- At phase $3 \pi / 2$, the pocket of hot gas is convected by the mean flow and the pressure within the chamber decreases, while the velocity perturbations of the dilution jets are now again very small.

As recalled in the introduction, each interaction between hot or cold pocket of gas and the nozzle generates acoustics which may propagate downstream (generating what is known as indirect noise) or upstream (generating another perturbation of the flame 

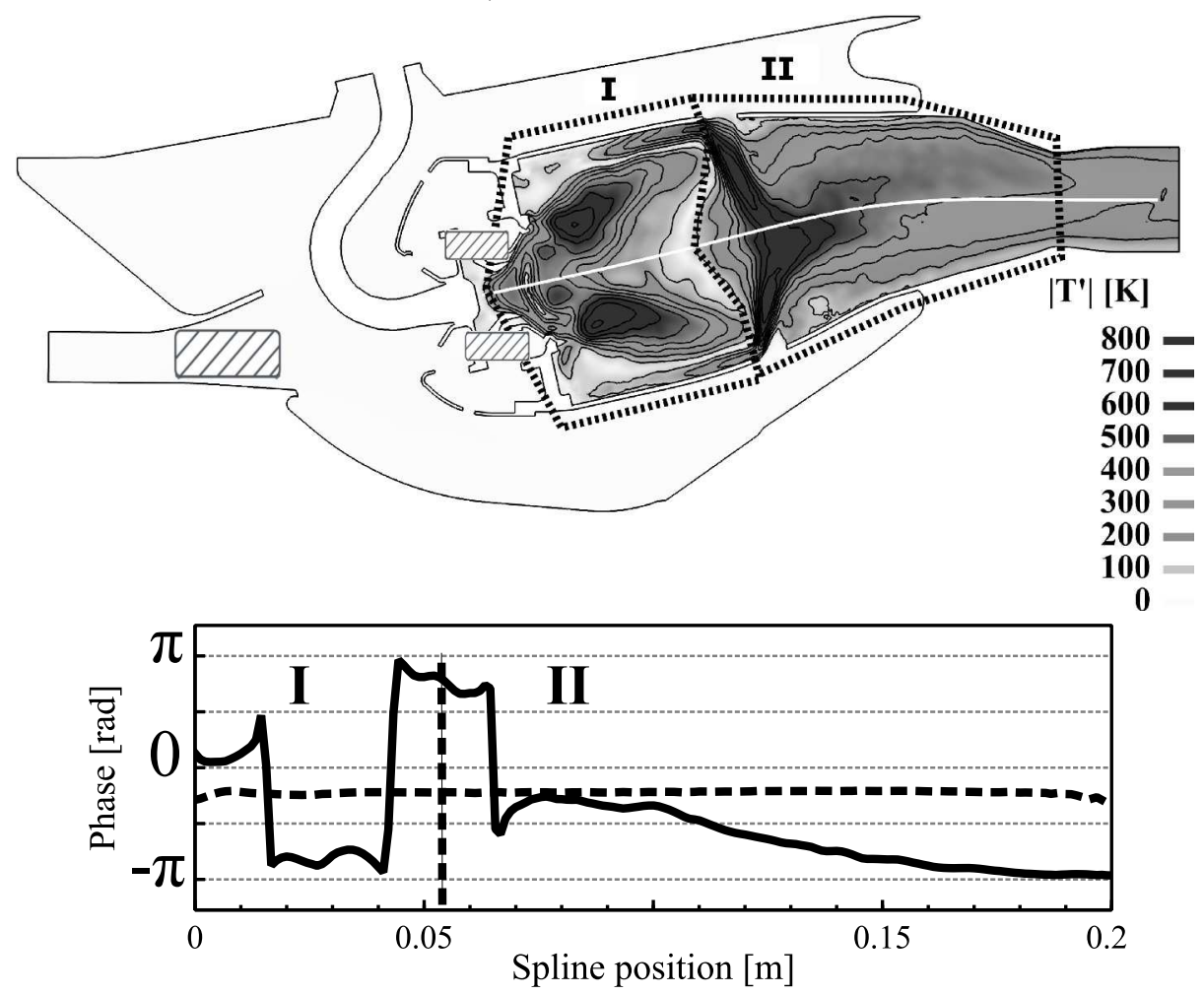

Figure 13. Temperature fluctuations for the first DMD mode at $323 \mathrm{~Hz}$ of operating point Cold. Top: Modulus over the medium plane. Bottom: phase of the temperature $(-)$ and pressure $(\cdots \cdots)$ along the path depicted by the white line.

region and promoting the creation of a new entropy spot). This creates a pressure drop between the surrounding casing and the combustor. In the same phase relation, depending on the sign of the pressure drop, the dilution jets are either: (a) flowing fresh air into the combustor, decreasing then the combustion and creating the cold pocket, (b) or blowing fresh air out of the combustor, enhancing the formation of a hot pocket. When the pressure drop decreases to zero, the dilution jets are annihilated and the pocket of cold/hot temperature is released and convected by the mean flow.

The acoustic response of the nozzle to an impacting temperature fluctuation can be characterised by the analytical theory based on the Euler equations of Duran \& Moreau (2013). The amplitude of the reflected acoustic wave scales with the incident temperature fluctuation and the ratio $\hat{p} / \hat{T}$ is typically of the order of $100 \mathrm{~Pa} / \mathrm{K}$ for most nozzles. As a consequence, for temperature fluctuations of approx. $350 \mathrm{~K}$ measured at $323 \mathrm{~Hz}$ from the DMD analysis of the LES data (see Fig. 14), the pressure fluctuations generated by the nozzle can then be computed with the help of the reflection coefficient and qualitatively estimated to be approx. $35000 \mathrm{~Pa}$, which is in fair agreement with the pressure fluctuations (see Fig. 11) measured in the LES. The angle of $\hat{p} / \hat{T}$ is between $3 \pi / 4$ and $\pi$, which is also in agreement with the LES/DMD results (see Fig. 13). Hence, for this nozzle and flow regime, temperature pockets impacting the nozzle generate strong opposite-sign pressure fluctuations in the combustion chamber. Note that any acoustic pressure fluctuation generates an amount of temperature fluctuations, even in absence of 

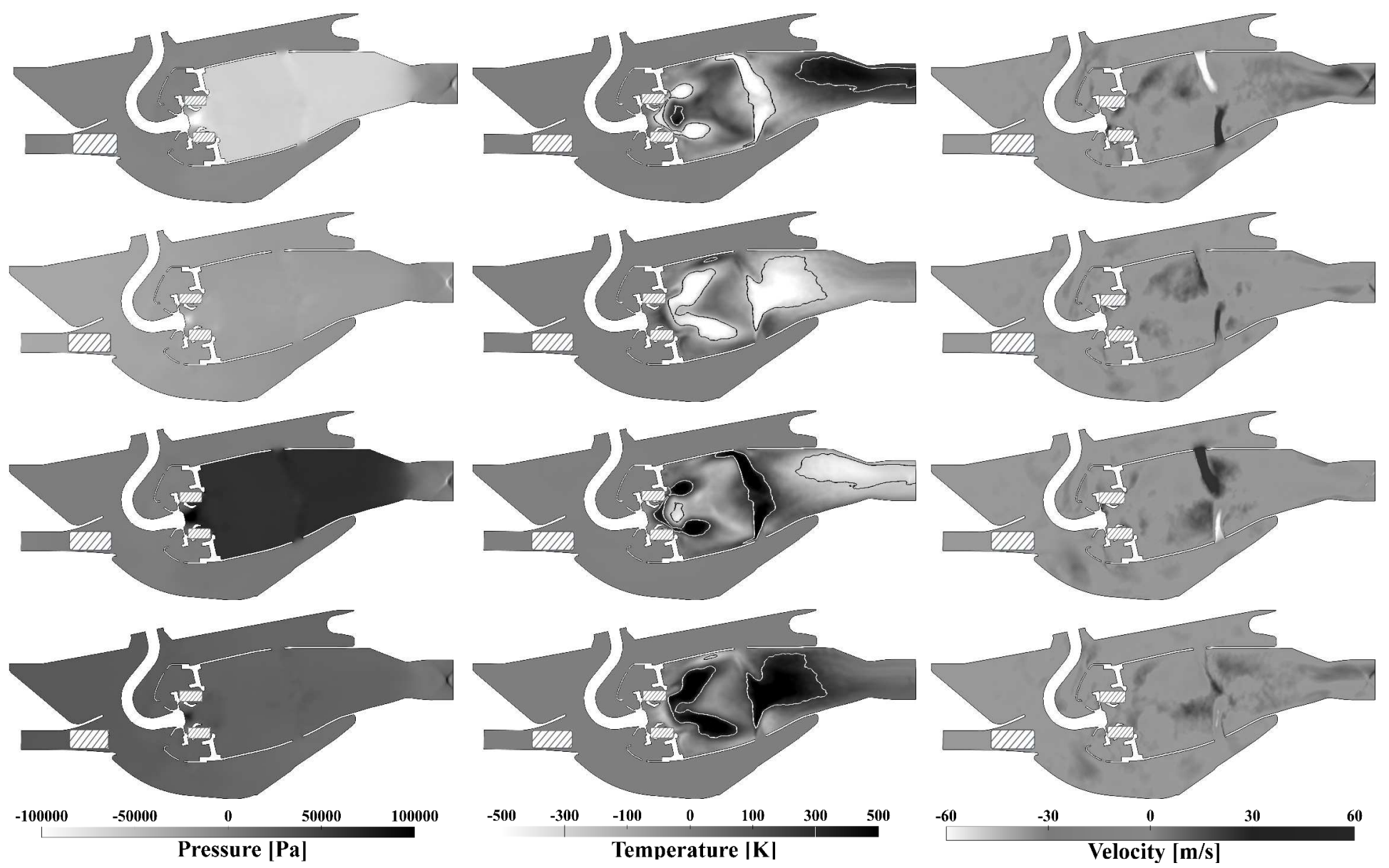

FigURE 14. Fluctuating pressure (left), temperature (middle) and velocity (right) from the DMD mode at $323 \mathrm{~Hz}$ for the operating point Cold $\left(T_{\text {inlet }}=557 \mathrm{~K}\right)$. From top to bottom, the four rows correspond to phases $0, \pi / 2, \pi$ and $3 \pi / 2$. 


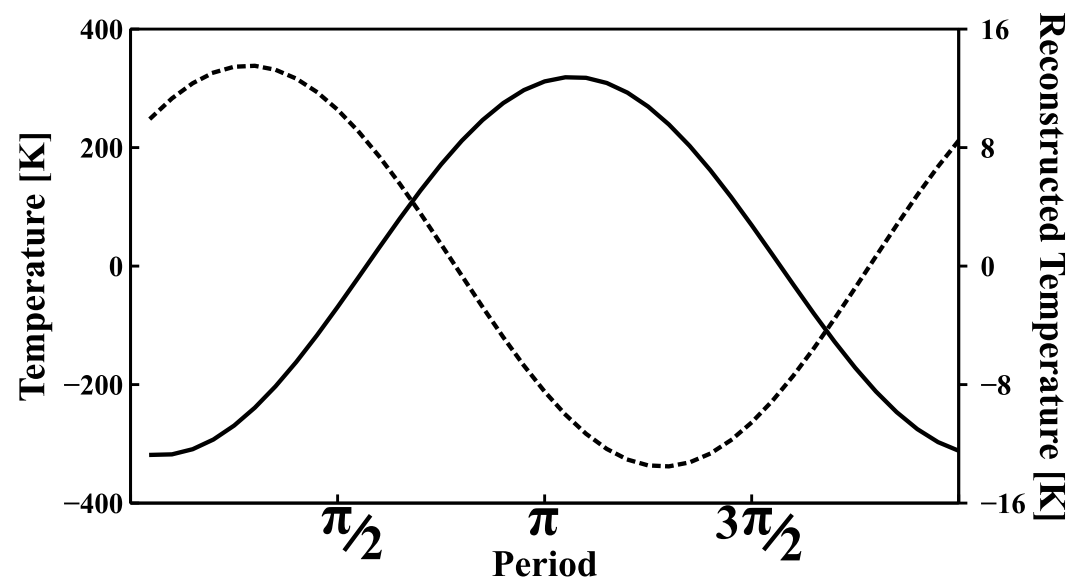

FiguRE 15. Solid line (-): reconstructed temperature signal at the entrance of the nozzle measured from the first DMD mode at $323 \mathrm{~Hz}$. Dotted line $(\cdots \cdots)$ : reconstructed acoustic temperature signal at the entrance of the nozzle computed with Eq. (3.17) and the pressure signal measured from the first DMD mode at $323 \mathrm{~Hz}$. Note that the second curve is multiplied by a factor 25 to be comparable to the first one.

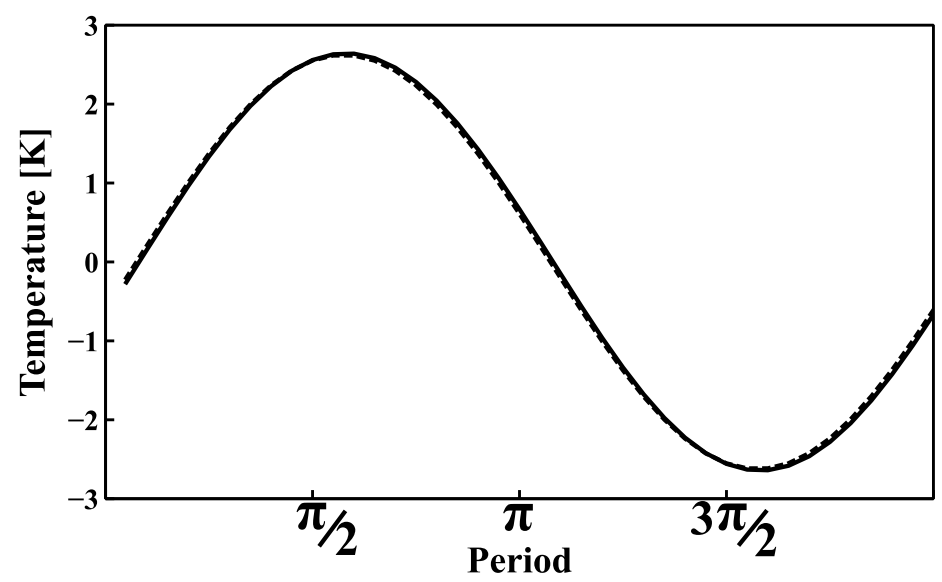

Figure 16. Solid line (-): reconstructed temperature signal at the air inlet entrance of the casing measured from the first DMD mode at $323 \mathrm{~Hz}$. Dotted line ( .......): reconstructed acoustic temperature signal at the air inlet entrance of the casing computed with Eq. (3.17) and the pressure signal measured from the first DMD mode at $323 \mathrm{~Hz}$.

nozzle or accelerated flow. Under the isentropic assumption one obtains:

$$
\hat{T}=\bar{T}\left(\frac{\bar{\gamma}-1}{\bar{\rho} \bar{c}^{2}}\right) \hat{p}
$$

Figure 15 shows that the isentropic temperature fluctuations at the entrance of the nozzle are almost 25 times smaller that the temperature fluctuations measured in the LES via the DMD and are also out of phase. This demonstrates that the temperature fluctuations seen in zone II are not the thermal signature of the pressure (acoustics) fluctuations in the chamber; instead they are generated by the primary zone/dilution jets interaction. After interacting with the nozzle, they generate a strong pressure fluctuation of opposite sign. This demonstrates that the combustion instability does not rely on a purely acoustic mode. Additionally, the temperature signal at the air inlet entrance in the 


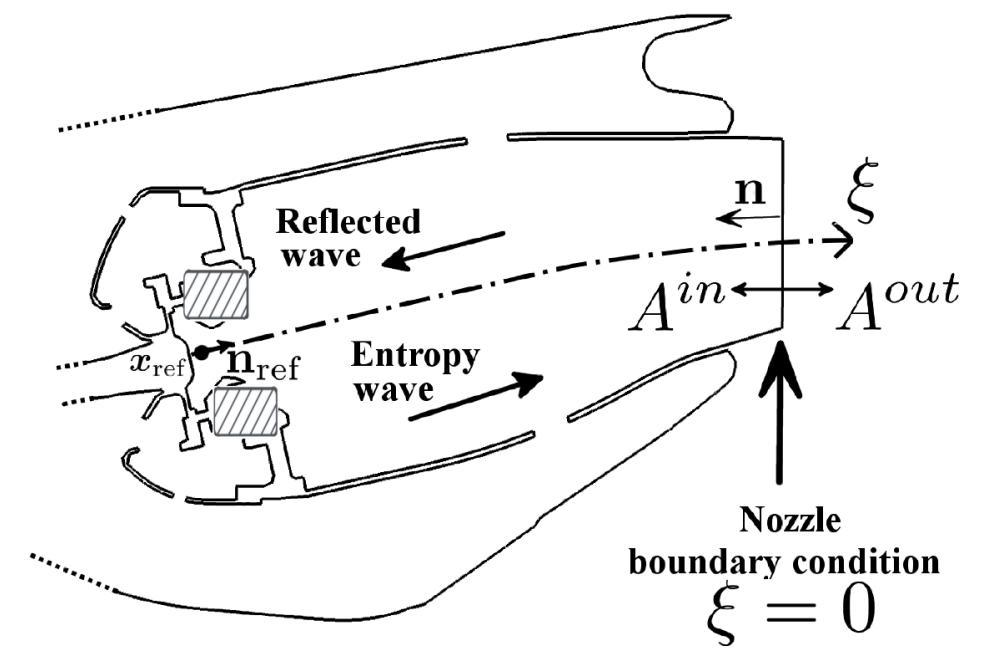

FIGURE 17. Schematic representation of the curvilinear coordinates system of the computational domain. The nozzle boundary condition is located at $\xi=0$. The normal $\mathbf{n}$ of the boundary is taken inward.

casing is also reconstructed from the DMD mode at $323 \mathrm{~Hz}$ and compared to the acoustic temperature signal. Results depicted in Fig. 16 suggest that the casing surrounding the combustion chamber features only a purely acoustic activity, demonstrating that the instability mechanism is confined in the combustion chamber and is not introduced at the air inlet of the domain. The idea that the unstable mode close to $320 \mathrm{~Hz}$ involves a coupling between entropy and acoustics is further supported by the numerical Helmholtz analysis of the combustor performed at Sec. 2.4.2, which shows that the smallest purely thermoacoustic frequency mode is close to $690 \mathrm{~Hz}$, very far from the observed $320 \mathrm{~Hz}$. The next two sections describe how this acoustic-entropy coupling phenomenon can be accounted for within the Helmholtz framework.

\section{Introducing entropy-acoustic coupling in Helmholtz formulation}

As detailed in previous works by the authors (see Motheau et al. 2013, 2014), the purely acoustic formulation used in Helmholtz solvers can be augmented to account simply for convective/acoustic modes by:

- modelling the entropy wave from the flame zone to the nozzle using a time delay (similar to flame transfer function delays used for classical thermoacoustics),

- introducing the entropy wave reflection into acoustics at the nozzle location by using an extended outlet impedance accounting for entropy/acoustic reflection.

These formulations are described in Sec. 4.1 which presents the Delayed Entropy Coupled Boundary Conditions (DECBC) model and tested in Sec. 4.2.

\subsection{Delayed entropy coupled boundary conditions}

The acoustic impedance is imposed to the computational domain as a uniform boundary condition over a discretised surface. In this context the boundary condition relies on a quasi-1D formulation. Hence, the fluctuating pressure and velocity fields can be reformulated in a general manner with the following 1D relations (Pierce 1981):

$$
\frac{\hat{p}(\xi)}{\gamma \bar{p}}=A^{\text {out }} e^{j k^{\text {out }} \xi}+A^{i n} e^{-j k^{i n} \xi}
$$




$$
\frac{\hat{\mathbf{u}}(\xi) \cdot \mathbf{n}}{\bar{c}}=-\left(A^{\text {out }} e^{j k^{\text {out }} \xi}-A^{\text {in }} e^{-j k^{i n} \xi}\right)
$$

where $k^{i n}=\omega / \bar{c}(1-M)$ and $k^{\text {out }}=\omega / \bar{c}(1+M)$ are the acoustic wave number of the acoustic waves $A^{\text {in }}$ and $A^{\text {out }}$ propagating along on the curvilinear axis $\xi$ (see Fig. 17) at the speed of sound $\bar{c}$ against or toward the direction of the flow, respectively. $A^{\text {in }}$ is the wave entering into the computational domain while $A^{\text {out }}$ is the wave leaving the domain at $\bar{c}(1+M)$. Note that the minus sign in front of the RHS of Eq. (4.2) stems from the fact that the unit vector normal to the exit boundary is collinear with the $\xi$-axis, pointing toward the negative $\xi$. Following Marble \& Candel (1977) an acoustic wave is generated by the entropy spots flowing through the exit nozzle or turbine. At the location $\xi=0$ where the boundary condition is imposed, the wave $A^{\text {in }}$ represents the acoustics generated by the nozzle and entering the computational domain. It can be assumed linear in the two outgoing waves:

$$
A^{\text {in }}=R_{u p} A^{\text {out }}+R_{\text {sp }} \sigma^{\text {out }}
$$

where $R_{u p}$ and $R_{s p}$ are the reflection coefficients for an impacting acoustic and entropy wave, respectively. The term $\sigma$ is the wave of the entropy fluctuations $\hat{s}(\xi) / C_{p}=\sigma^{o u t} e^{j k_{s} \xi}$ that is convected at the mean flow speed and leaving the domain, $C_{p}$ is the heat capacity at constant pressure and $k_{s}=\omega /(\bar{c} M)$ the wave number associated to the convection of entropy spots by the mean flow. At $\xi=0$, Eqs. (4.1) and (4.2) become:

$$
\begin{array}{r}
\frac{\hat{p}(\xi)}{\gamma \bar{p}}=A^{\text {out }}+A^{\text {in }} \\
\frac{\hat{\mathbf{u}}(\xi) \cdot \mathbf{n}}{\bar{c}}=-A^{\text {out }}+A^{\text {in }}
\end{array}
$$

while

$$
\frac{\hat{s}}{C_{p}}=\sigma^{\text {out }}
$$

Eq. (4.3) may then be recast into the following relation:

$$
\hat{p}\left(R_{u p}-1\right)-\bar{\rho} \bar{c} \hat{\mathbf{u}} \cdot \mathbf{n}\left(R_{u p}+1\right)+2 \frac{\overline{\gamma p}}{C_{p}} R_{s p} \hat{s}=0
$$

with $R_{u p}=A^{\text {in }} / A^{\text {out }}$ and $R_{s p}=A^{\text {in }} / \sigma^{\text {out }}$. Note that all the parameters $\bar{\gamma}, \bar{p}, \bar{\rho}, \bar{c}$ and $C_{p}$ must be taken at the location where the boundary condition represented by Eq. (4.7) is imposed. After introducing the relation between the acoustic impedance and the acoustic reflection coefficient $Z_{u p}^{0}=\left(R_{u p}+1\right) /\left(R_{u p}-1\right)$, the implementation of Eq. (4.7) into the Helmholtz solver AVSP leads finally to the following reformulation of the boundary condition (4.7):

$$
\frac{\hat{p}}{Z_{u p}^{0}}-\bar{\rho} \bar{c} \hat{\mathbf{u}} \cdot \mathbf{n}+\frac{Z_{u p}^{0}-1}{Z_{u p}^{0}} \frac{\overline{\gamma p}}{C_{p}} R_{s p} \hat{s}=0
$$

Note that similarly to the impedance $Z_{u p}^{0}, R_{s p}$ can be deduced either by the compact hypothesis of Marble \& Candel (1977) or the analytical LEE method of Duran \& Moreau (2013).

As the Helmholtz solver relies on the zero-Mach-number assumption, the effect of elements, such as the nozzle, where a mean flow occurs can simply be taken into account by imposing the right energy flux through the proper impedance formulation (see Sec. 2.4.1). However imposing only the acoustic impedance, Eq. (2.6), means neglecting the entropy-acoustics coupling and the subsequent sound being generated by the entropy spots flowing through the exit nozzle or turbine. 


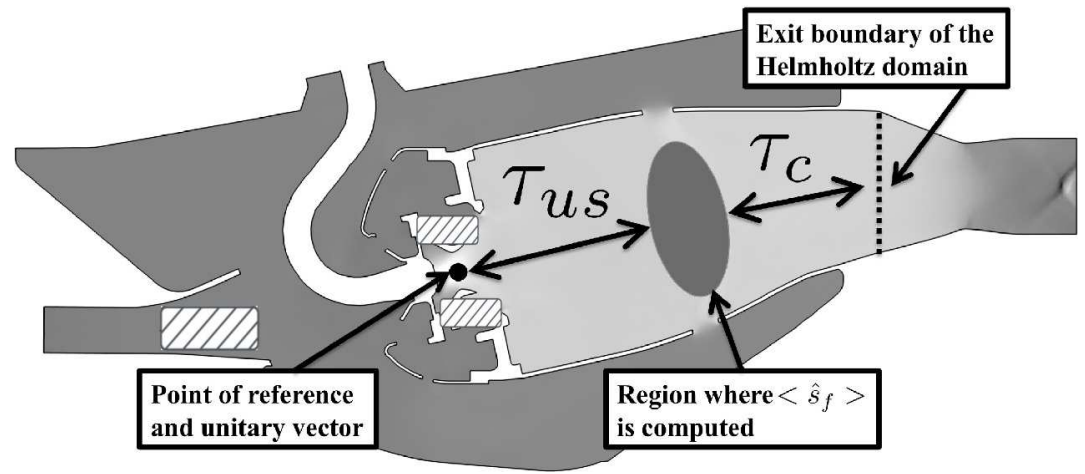

Figure 18. Computational domain for the Helmholtz analysis. The point of reference and the zone of averaging used for the entropy generation modelling (Eq. 4.11) are displayed as well as the exit section where the entropy-acoustic boundary condition (Eq. 4.10) is applied.

Now, $\hat{s}$ in Eq. (4.8) is not available in the Helmholtz domain and the entropy fluctuation at the edge of the combustion chamber must be modeled before Eq. (4.8) can be applied. Assuming that the entropy fluctuations at the exit have first been generated in the flame region before being convected by the mean flow, one obtains $\hat{s}=\hat{s}_{f} \exp \left(j \omega \tau_{c}\right)$ where $\hat{s}_{f}$ is the amount of entropy being generated by the flame and $\tau_{c}$ is the convection time from the flame to the exit. Consistently with the Helmholtz framework, it is then useful to relate the generated entropy $\hat{s}_{f}$ to some acoustic quantity. It is done here in a way similar to the $n-\tau$ model (Crocco 1952) for unsteady heat release, connecting the entropy fluctuation to the acoustic velocity taken at a reference point located upstream of the flame region :

$$
\hat{s}_{f}=G_{\mathrm{us}} e^{j \omega \tau_{\mathrm{us}}} \hat{\mathbf{u}}_{\mathrm{ref}} \cdot \mathbf{n}_{\mathrm{ref}}
$$

where $G_{\mathrm{us}}$ and $\tau_{\mathrm{us}}$ are the gain and the time delay of the entropy generation from an acoustic perturbation $\hat{\mathbf{u}}$ at the location $\boldsymbol{x}_{\text {ref }}$ and along the direction $\mathbf{n}_{\text {ref }}$. Rewriting of Eq. (4.8) then leads to the following Delayed Entropy Coupled Boundary Condition (DECBC):

$$
\frac{\hat{p}}{Z_{u p}^{0}}-\bar{\rho} \bar{c} \hat{\mathbf{u}} \cdot \mathbf{n}+\frac{Z_{u p}^{0}-1}{Z_{u p}^{0}} \frac{\overline{\gamma p}}{C_{p}} R_{s p} G_{\mathrm{us}} e^{j \omega \tau_{\mathrm{us}}} e^{j \omega \tau_{c}} \hat{\mathbf{u}}_{\mathrm{ref}} \cdot \mathbf{n}_{\mathrm{ref}}=0
$$

The Delayed Entropy Coupled Boundary Condition (DECBC) has been validated elsewhere in the case of an academic 1D combustor mounted on a compact nozzle ((see Motheau et al. 2012, 2014)).

\subsection{Application of the DECBC approach}

Recall that the configuration investigated in the present paper exhibits a low frequency mode of oscillation at a frequency significantly smaller than any acoustic mode. Helmholtz analysis performed in Sec. 2.4.2 failed to capture this low frequency mode. It is thus natural to investigate whether the DECBC approach described in Sec. 4.1 helps to recover this latter mode. The transfer function between the acoustic velocity upstream of the flame and the entropy generated downstream of the primary zone was assessed by postprocessing the LES via the DMD analysis. More precisely, this entropy transfer function was defined as

$$
G_{\mathrm{us}}^{\mathrm{LES}} e^{j \omega \tau_{\mathrm{us}}^{\mathrm{LES}}}=\frac{<\hat{s}_{f}>}{\hat{\mathbf{u}}_{\mathrm{ref}} \cdot \mathbf{n}_{\mathrm{ref}}}
$$




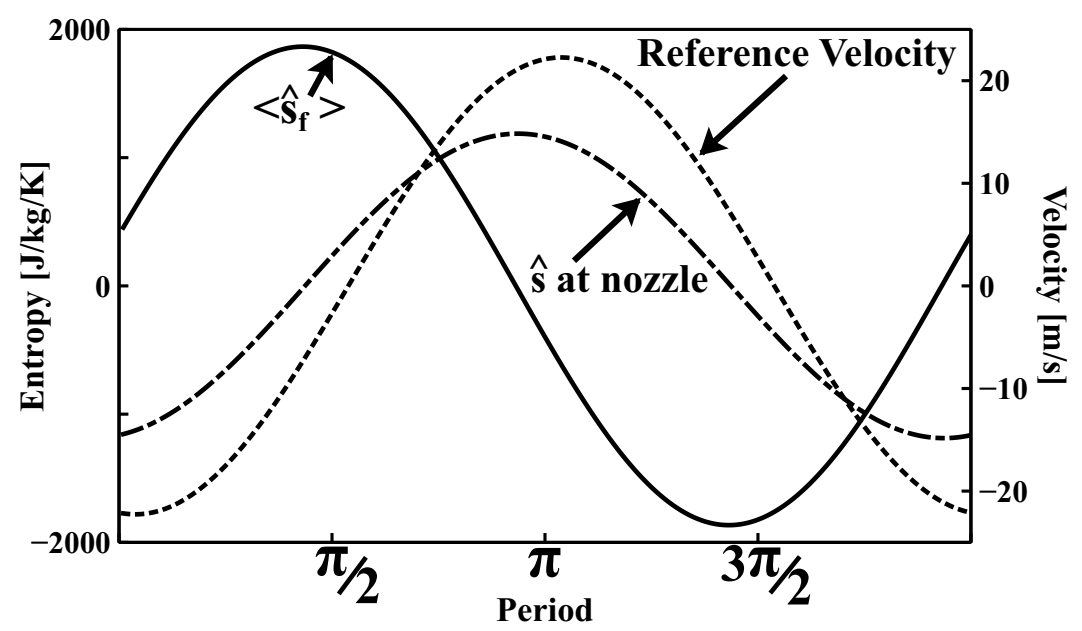

FIGURE 19. Reconstructed signals from the first DMD mode at $323 \mathrm{~Hz}$ for the operating point Cold. Dotted line $(\cdots \cdots)$ : reference velocity $\hat{\mathbf{u}}_{\text {ref }} \cdot \mathbf{n}_{\text {ref }}$. Solid line $(-)$ : entropy $\left\langle\hat{s}_{f}\right\rangle$ averaged in the middle of the combustor. Dot-dashed line $(---)$ : entropy $\langle\hat{s}\rangle$ averaged at the exit plane upstream of the nozzle.

where $\mathbf{n}_{\text {ref }}$ is a unitary vector of reference aligned with the main axis of the combustor (as in Sec. 2.4, Fig. 10), $\hat{\mathbf{u}}_{\text {ref }}$ is the acoustic velocity at the reference point depicted in Fig. 18 and $\left\langle\hat{s}_{f}\right\rangle$ is the entropy fluctuation averaged over a small volume located downstream of the primary combustion zone (see Fig. 18), in agreement with the mode structure observed from the DMD analysis of Sec. 3.2. Obtaining the acoustic velocity fluctuations $\hat{\mathbf{u}}_{\text {ref }}$ is challenging because the reference point is located inside the swirler where hydrodynamic fluctuations are present. However, as the present study focuses on a low-frequency instability, the hydrodynamic component is considered negligible. Note that this transfer function is similar to but different from the classical flame transfer function which relates the upstream acoustic velocity to the unsteady heat release based on a $n-\tau$ type of model (Crocco 1952; Nicoud et al. 2007). Consequently, the fluctuating velocity $\hat{\mathbf{u}}_{\text {ref }}$ for both the active flame and DECBC approach is chosen at the same reference point. Following the rationale developed in Sec. 4.1, the DMD analysis was also used to measure the convection time $\tau_{c}^{\text {LES }}$ from the flame region to the end of the combustion chamber (see Fig. 18). Note that because the entropy spots decay during their convection through the chamber exit (because of the turbulent mixing and dissipation), the time delay $\tau_{c}^{\mathrm{LES}}$ must be completed by a gain $G_{c}^{\mathrm{LES}}$ (smaller than unity) to relate the entropy in the flame region to the entropy at the exit:

$$
\hat{s}=G_{c}^{\mathrm{LES}} e^{j \omega \tau_{c}^{\mathrm{LES}}}<\hat{s}_{f}>
$$

Finally, the entropy fluctuations in Eq. (4.8) can be modeled as :

$$
\hat{s}=G_{\mathrm{us}}^{\mathrm{LES}} G_{c}^{\mathrm{LES}} e^{j \omega\left(\tau_{\mathrm{us}}^{\mathrm{LES}}+\tau_{c}^{\mathrm{LES}}\right)} \hat{\mathbf{u}}_{\mathrm{ref}} \cdot \mathbf{n}_{\mathrm{ref}}
$$

The DECBC condition for the 3D SAFRAN combustor is obtained by injecting Eq. (4.13) into (4.10) and reads:

$$
\frac{\hat{p}}{Z_{u p}^{0}}-\bar{\rho} \bar{c} \hat{\mathbf{u}} \cdot \mathbf{n}+\frac{Z_{u p}^{0}-1}{Z_{u p}^{0}} \frac{\overline{\gamma p}}{C_{p}} R_{s p} G_{\mathrm{us}}^{\mathrm{LES}} G_{c}^{\mathrm{LES}} e^{j \omega\left(\tau_{\mathrm{us}}^{\mathrm{LES}}+\tau_{c}^{\mathrm{LES}}\right)} \hat{\mathbf{u}}_{\mathrm{ref}} \cdot \mathbf{n}_{\mathrm{ref}}=0
$$

Note that the fields of $n_{\text {local }}$ and $\tau_{\text {local }}$ for the active flame (see Eq. (2.3)) have also 
Mixed acoustic-entropy combustion instabilities in gas turbines

\begin{tabular}{c|cc|cc} 
Operating Point & $G_{\mathrm{us}}^{\mathrm{LES}}$ & $\tau_{\mathrm{us}}^{\mathrm{LES}}[\mathrm{s}]$ & $G_{c}^{\mathrm{LES}}$ & $\tau_{c}^{\mathrm{LES}}[\mathrm{s}]$ \\
\hline Cold $\left(T_{\text {inlet }}=557 \mathrm{~K}\right)$ & 84 & $2.13 \times 10^{-3}$ & 0.63 & $7.82 \times 10^{-4}$ \\
$\operatorname{Hot}\left(T_{\text {inlet }}=762 \mathrm{~K}\right)$ & 74 & $1.76 \times 10^{-3}$ & 0.48 & $4.14 \times 10^{-4}$
\end{tabular}

TABLE 4. Gain and time delay of the transfer functions modelling the entropy fluctuations for the operating points Cold $\left(T_{\text {inlet }}=557 \mathrm{~K}\right)$ and $H o t\left(T_{\text {inlet }}=762 \mathrm{~K}\right)$.

\begin{tabular}{c|c|c} 
Operating Point & Frequency $[\mathrm{Hz}]$ & Growth/Decay rate $\left[\mathrm{s}^{-1}\right]$ \\
\hline Cold $\left(T_{\text {inlet }}=557 \mathrm{~K}\right)$ & 318 & +267 \\
Hot $\left(T_{\text {inlet }}=762 \mathrm{~K}\right)$ & 429 & +12
\end{tabular}

TABLE 5. Helmholtz computations with the DECBC approach.

been computed from the DMD analysis and it has been checked that they are very close to the one computed with Fourier Transform (see Sec. 2.4.2). The first DMD mode at $323 \mathrm{~Hz}($ Cold $)$ was also used to assess the entropy related gains $G_{\text {us }}^{\mathrm{LES}} ; G_{c}^{\mathrm{LES}}$ and time delays $\tau_{\text {us }}^{\mathrm{LES}} ; \tau_{c}^{\mathrm{LES}}$. Figure 19 presents the following reconstructed signals from this DMD mode:

- the reference fluctuating velocity $\hat{\mathbf{u}}_{\text {ref }} \cdot \mathbf{n}_{\text {ref }}$

- the fluctuating entropy $\left\langle\hat{s}_{f}>\right.$ averaged over a volume located in the middle of the combustor where the formation of the pocket occurs (see Fig. 18)

- the fluctuating entropy $\hat{s}$ averaged over the exit boundary condition of the Helmholtz domain.

The gain and time-delay of transfer functions described in Eqs. (4.11) and (4.12) can therefore be easily estimated with the help of elementary signal processing. Results are summarised in Table 4 for the two operating points Cold $\left(T_{\text {inlet }}=557 \mathrm{~K}\right)$ and Hot $\left(T_{\text {inlet }}=762 \mathrm{~K}\right)$. Note that these values are supposed independent on frequency.

\subsection{Helmholtz/DECBC results}

Helmholtz computations are performed for each operating point Cold and Hot by using the respective fields of sound speed, active flame $n-\tau$ fields and transfer functions from Table 4 . The outlet impedance $Z_{m J}^{M}$ is computed from the Duran \& Moreau (2013) theory. Results for the first mode are gathered in Table 5. As already stated in Sec. 2.4.2, the first acoustic mode for the operating point Cold oscillates at around $690 \mathrm{~Hz}$, very far from the $320 \mathrm{~Hz}$ instability observed in the LES and experiment; this acoustic mode is slightly damped (decay rate of approximatively $-40 s^{-1}$ ), even while taking into account the proper acoustic flux at the inlet/outlet as well as the coupling between acoustics and combustion through the active flame model (see Table 2). Introducing the DECBC approach corrects this behaviour: the Helmholtz solver now gives a strong unstable mode (growth rate $267 \mathrm{~s}^{-1}$ ) oscillating at $318 \mathrm{~Hz}$ (see Table 5), in fair agreement with the LES and experiment. Note that the frequency of oscillation obtained $(318 \mathrm{~Hz})$ is rather close to the first DMD mode $(323 \mathrm{~Hz})$, thus justifying a posteriori the fact that the transfer function gain and delay were supposed independent on frequency when performing the Helmholtz computations. The shapes of the first modes from the DMD analysis and the Helmholtz computations with the DECBC approach are depicted in Fig. 20. The fluctuating pressure $\hat{p}$ is made non dimensional by setting $\max (\Re(\hat{p}))=1$. It is remarkable that the eigenmode computed by the Helmholtz solver with the DECBC approach (bottom of Fig. 20) is rather similar to the DMD mode (top of Fig. 20). The maximum amplitude of fluctuation is located inside the whole combustion chamber, while the casing exhibits a structure close to a quarter wave mode. 

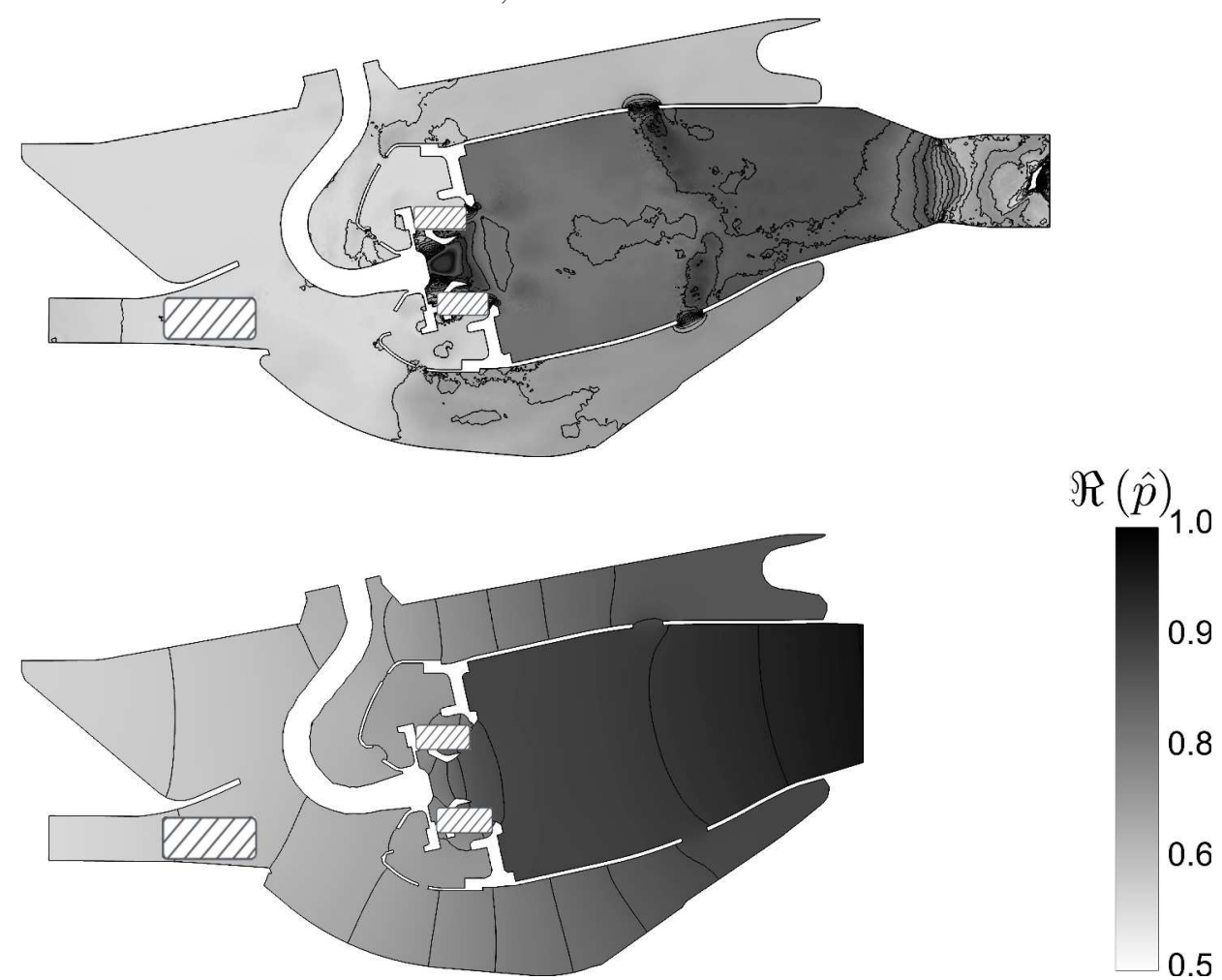

FIGURE 20. Shapes of the first pressure eigenmode for the operating point Cold. Top: DMD analysis $(323 \mathrm{~Hz})$. Bottom: Helmholtz computation with the DECBC approach $(318 \mathrm{~Hz})$. The fluctuating pressure $\hat{p}$ is made non dimensional by setting $\max (\Re(\hat{p}))=1$.

The methodology described previously is also applied to the operating point Hot. Results exhibit the same behaviour (Table 5). A mode is found at $429 \mathrm{~Hz}$ with a small growth rate, consistent with the LES result which showed a small amplitude mode at $435 \mathrm{~Hz}$. In Silva et al. (2013), authors mention that the growth rates assessed by a Helmholtz solver should be offset by a negative quantity which represents all the damping effects not included in the zero-Mach-number framework (acoustic boundary layers, vortex generation in shear layers, etc.). Consequently the mode predicted for case Hot at $\left(+12 \mathrm{~s}^{-1}\right)$ is consistent with the DMD analysis and experiments. Note also that the growth rate obtained for Cold is much larger than for Hot in full agreement with the LES/experimental results which display a strong instability in the first case only. The acoustic-entropy coupling allows, once included in a low-order Helmholtz solver, to recover the low frequency instability of the LES/experiments. This demonstrates that this instability is indeed driven by the acoustic waves generated by strong entropy fluctuations being convected through the nozzle. To the authors knowledge, this is the first time that such an instability is identified in an aeroengine configuration.

\section{Conclusion}

High-fidelity computations of a realistic aerocombustor that features a strong lowfrequency instability under certain operating conditions have been performed. Experiments show that the establishment of an instability depends to first order on the inlet temperature of the air entering the combustion chamber. Large Eddy Simulations of two 
operating points representative of the stable/unstable states of the combustor were first performed, showing that the computations can discriminate the stable or unstable operating points. In any case a low frequency mode is found, featuring either strong or very weak fluctuations of all physical variables. The DMD analysis demonstrates that pockets of fluctuating entropy/temperature are created inside the combustor and convected to the downstream nozzle, creating a reflected acoustic perturbation that goes back to the flame.

The LES were complemented by a purely acoustic analysis where the coupling between acoustics and combustion is taken into account by an active flame model based on a $n-\tau$ formalism. For both operating points, results exhibit a damped eigenmode which frequency is very far from the low-frequency mode observed in the experiments and LES computations. This suggests that the mechanism leading to the combustion instability relies on a coupling between entropy and acoustic waves inside the nozzle; as this latter mechanism is neglected in the Helmholtz analysis due to the zero-Mach-number assumption, the low-frequency mode can not be reproduced.

A Delayed Entropy Coupled Boundary Condition (DECBC) is then developed as a mean to recover some of the convective effects when representing a thermo-acoustic system under the zero-Mach-number formalism. This model consists of two steps, one for the entropy generation in the flame region, a second one for the convection/dissipation of the entropy spots through the combustion chamber. This results in a transfer function which relates a reference acoustic velocity in the burner and entropy at the exit of the chamber and which was assessed by post-processing LES data via the DMD analysis. The acoustics generated by the convection of the entropy spots through the exit nozzle are then treated by applying a proper boundary condition which couples entropy and acoustic quantities.

Helmholtz computations coupled with the DECBC approach are conducted for both stable/unstable operating points. Results exhibit low-frequency eigenmodes with frequency, shape and stability behaviour in very good agreement with the experimental and LES data. This enlightens the major role played by the coupling between entropy and acoustic waves inside the nozzle on the establishment of this combustion instability, contrary to the coupling between the flame and the acoustics or the sound speed field which have a minor influence. This study shows that the acoustic/entropy coupling should be accounted for when performing the thermo-acoustic analysis of (complex) combustors, contrary to what is usually done.

E. Motheau gratefully acknowledges support from SNECMA. The authors also thank T. Jaravel (CERFACS) for the technical support for the DMD analysis as well as Y. Méry (SNECMA) for providing the data and the fruitful discussions about the configuration. Part of this study has been performed during the Summer Program 2012 of the Center for Turbulence Research, Stanford University, USA.

\section{REFERENCES}

Abouseif, G. E., Keklak, J. A. \& Toong, T. Y. 1984 Ramjet rumble: The low-frequency instability mechanism in coaxial dump combustors. Combust. Sci. Tech. 36 (1-2), 83-108.

Bake, F., Richter, C., Muhlbauer, B., Kings, N., Rohle, I., Thiele, F. \& Noll, B. 2009 The entropy wave generator (ewg): a reference case on entropy noise. J. Sound Vib. pp. $574-598$.

Bell, W., Daniel, B. \& Zinn, B. 1973 Experimental and theoretical determination of the admittances of a family of nozzles subjected to axial instabilities. J. Sound Vib. $30(2)$, 179-190. 
Bloy, A. W. 1979 The pressure waves produced by the convection of temperature disturbances in high subsonic nozzle flows. J. Fluid Mech. 94, 465-475.

Bodony, D. J. 2009 Scattering of an entropy disturbance into sound by a symmetric thin body. Phys. Fluids 21 (9), 096101.

Boнn, M.S. 1977 Response of a subsonic nozzle to acoustic and entropy disturbances. J. Sound Vib. $\mathbf{5 2}(2), 283-297$.

Candel, S., Durox, D., Ducruix, S., Birbaud, A.L., Noiray, N. \& Schuller, T. 2009 Flame dynamics and combustion noise : progress and challenges. Int. J. Aeroacoustics $\mathbf{8}$, $1-56$.

CERFACS 2009 AVBP Handbook - http://cerfacs.fr/ avbp/AVBP_V5.X/HANDBOOK. CERFACS.

Chedevergne, F., Casalis, G. \& Majdalani, J. 2012 Direct numerical simulation and biglobal stability investigations of the gaseous motion in solid rocket motors. J. Fluid Mech. 706, 190-218.

Chen, K. K., Tu, J. H. \& Rowley, C. W. 2012 Variants of dynamic mode decomposition: Boundary condition, koopman, and fourier analyses. Journal of Nonlinear Science 22 (6), 887-915.

Colin, O., Ducros, F., Veynante, D. \& Poinsot, T. 2000 A thickened flame model for large eddy simulations of turbulent premixed combustion. Phys. Fluids 12 (7), 1843-1863.

Colin, O. \& Rudgyard, M. 2000 Development of high-order taylor-galerkin schemes for unsteady calculations. J. Comput. Phys. 162 (2), 338-371.

Crocco, L. 1952 Aspects of combustion instability in liquid propellant rocket motors. part II. J. American Rocket Society 22, 7-16.

Culick, F. E. C. \& Kuentzmann, P. 2006 Unsteady Motions in Combustion Chambers for Propulsion Systems. NATO Research and Technology Organization.

Cumpsty, N. A. \& Marble, F. E. 1977 The interaction of entropy fluctuations with turbine blade rows; a mechanism of turbojet engine noise. Proc. R. Soc. Lond. A 357, 323-344.

DoAK, P.E. 1998 Fluctuating total enthalpy as the basic generalized acoustic field. Theoret. Comput. Fluid Dynamics 10 (1), 115-133.

Dowling, A. P. \& Stow, S. R. 2003 Acoustic Analysis of Gas Turbine Combustors. J. Prop. Power 19 (5), 751-764.

Duran, I. \& Moreau, S. 2013 Solution of the quasi-one-dimensional linearized euler equations using flow invariants and the magnus expansion. J. Fluid Mech. 723, 190-231.

Duran, I., Moreau, S. \& Poinsot, T. 2013 Analytical and numerical study of combustion noise through a subsonic nozzle. AIAA Journal $\mathbf{5 1}$ (1), 42-52.

Eckstein, J., Freitag, E., Hirsch, C. \& Sattelmayer, T. 2006 Experimental study on the role of entropy waves in low-frequency oscillations in a rql combustor. J. Eng. Gas Turb. and Power 128 (2), 264-270.

Ffowcs-Williams, J. E. \& Howe, M. S. 1975 The generation of sound by density inhomogeneities in low mach number nozzle flows. J. Fluid Mech. 70 (03), 605-622.

Franzelli, B., Riber, E., Sanjosé, M. \& Poinsot, T. 2010 A two-step chemical scheme for Large-Eddy Simulation of kerosene-air flames. Combust. Flame 157 (7), 1364-1373.

Gicquel, L. Y. M., Staffelbach, G. \& Poinsot, T. 2012 Large eddy simulations of gaseous flames in gas turbine combustion chambers. Prog. Energy Comb. Sci. 38 (6), $782-817$.

Goh, C. S. \& Morgans, A. S. 2011 Phase prediction of the response of choked nozzles to entropy and acoustic disturbances. J. Sound Vib. 330 (21), 5184-5198.

Goh, C. S. \& Morgans, A. S. 2013 The influence of entropy waves on the thermoacoustic stability of a model combustor. Combustion Science and Technology 185 (2), 249-268.

Hield, P., Brear, M. \& Jin, S.H. 2009 Thermoacoustic limit cycles in a premixed laboratory combustor with open and choked exits. Combust. Flame pp. 1683-1697.

Hochgreb, S., Dennis, D. J. C., Ayranci, I., Bainbridge, W. \& Cant, S. 2013 Forced and self-excited instabilities from lean premixed, liquid-fuelled aeroengine injectors at high pressures and temperatures. In Proceedings of ASME Turbo Expo 2013 - GT2013-9531.

Howe, M. S. 2010 Indirect combustion noise. J. Fluid Mech. 659, 267-288.

Karimi, N., Brear, M.J., Jin, S.-H. \& Monty, J.P. 2009 Linear and non-linear forced response of a conical, ducted, laminar premixed flame. Combust. Flame pp. 2201-2212. 
Karlsson, M. \& Åвом, M. 2010 Aeroacoustics of t-junctions - an experimental investigation. J. Sound Vib. $329(10), 1793-1808$.

KELLER, J. J. 1995 Thermoacoustic oscillations in combustion chambers of gas turbines. AIAA Journal 33 (12), 2280-2287.

Keller, J. J., Egli, W. \& Hellat, J. 1985 Thermally induced low-frequency oscillations. Zeitschrift für angewandte Mathematik und Physik ZAMP 36, 250-274.

Kim, K. T., Lee, J. G., Quay, B. D. \& Santavicca, D. A. 2010 Response of partially premixed flames to acoustic velocity and equivalence ratio perturbations. Combust. Flame 157 (9), 1731 - 1744.

Kornilov, V., Rook, R., ten Thije Boonkkamp, J. \& De Goey, L. 2009 Experimental and numerical investigation of the acoustic response of multi-slit bunsen burners. Combust. Flame pp. 1957-1970.

Lawn, C. J., Evesque, S. \& Polifke, W. 2004 A model for the thermoacoustic response of a premixed swirl burner, part i: Acoustic aspects. Combust. Sci. Tech. 176 (8), 1331-1358.

Leyko, M., Moreau, S., Nicoud, F. \& Poinsot, T. 2011 Numerical and analytical modelling of entropy noise in a supersonic nozzle with a shock. J. Sound Vib. 330 (16, 1), 3944-3958.

Lieuwen, T. \& YAng, V. 2005 Combustion Instabilities in Gas Turbine Engines: Operational Experience, Fundamental Mechanisms, and Modeling. AIAA Prog. in Astronautics and Aeronautics.

Macquisten, M. A. \& Dowling, A. P. 1994 Low-frequency combustion oscillations in a model afterburner. Combust. Flame 94 (4), 253-264.

Marble, F. E. \& CAndel, S. 1977 Acoustic disturbances from gas nonuniformities convected through a nozzle. J. Sound Vib. 55, 225-243.

Martin, C., Benoit, L., Sommerer, Y., Nicoud, F. \& Poinsot, T. 2006 LES and acoustic analysis of combustion instability in a staged turbulent swirled combustor. AIAA Journal 44 (4), 741-750.

Mcmanus, K., Poinsot, T. \& Candel, S. 1993 A review of active control of combustion instabilities. Prog. Energy Comb. Sci. 19, 1-29.

Mendez, S. \& Nicoud, F. 2008 a Adiabatic homogeneous model for flow around a multiperforated plate. AIAA Journal 46 (10), 2623-2633.

Mendez, S. \& Nicoud, F. $2008 b$ Large-eddy simulation of a bi-periodic turbulent flow with effusion. J. Fluid Mech. 598, 27-65.

Miles, J. H. 2010 Separating direct and indirect turbofan engine combustion noise using the correlation function. Journal of propulsion and power 26 (5), 1144-1152.

Moase, W. H., Brear, M. J. \& Manzie, C. 2007 The forced response of choked nozzles and supersonic diffusers. J. Fluid Mech. 585, 281-304.

Morfey, C. L. 1973 Amplification of aerodynamic noise by convected flow inhomogeneities. J. Sound Vib. 31, 391-397.

Morgans, A. S., Goh, C. S. \& Dahan, J. A. 2013 The dissipation and shear dispersion of entropy waves in combustor thermoacoustics. J. Fluid Mech. 733 (R2).

Motheau, E., Méry, Y., Nicoud, F. \& Poinsot, T. 2013 Analysis and modeling of entropy modes in a realistic aeronautical gas turbine. J. Eng. Gas Turb. and Power 135 (9), 092602.

Motheau, E., Nicoud, F. \& Poinsot, T. 2012 Using boundary conditions to account for mean flow effects in a zero mach number acoustic solver. J. Eng. Gas Turb. and Power 134 (11), 111502.

Motheau, E., Selle, L. \& Nicoud, F. 2014 Accounting for convective effects in zero-machnumber thermoacoustic models. J. Sound Vib. 333 (1), $246-262$.

Nicoud, F., Baya Toda, H., Cabrit, O., Bose, S. \& Lee, J. 2011 Using singular values to build a subgrid-scale model for large eddy simulations. Phys. Fluids 23 (8), 085106.

Nicoud, F., Benoit, L., Sensiau, C. \& Poinsot, T. 2007 Acoustic modes in combustors with complex impedances and multidimensional active flames. AIAA Journal 45, 426-441.

Nicoud, F. \& WieczoreK, K. 2009 About the zero mach number assumption in the calculation of thermoacoustic instabilitie. Int. J. Spray and Combustion Dynamic 1, 67-112.

Peters, M., Hirschberg, A., Reijnen, A. J. \& Wijnands, A. P. J. 1993 Damping and reflection coefficient measurements for an open pipe at low mach and low helmholtz numbers. J. Fluid Mech. 256, 499-499. 
Pierce, A. D. 1981 Acoustics: an introduction to its physical principles and applications. New York: McGraw Hill.

Pitsch, H. 2006 Large eddy simulation of turbulent combustion. Ann. Rev. Fluid Mech. 38, 453-482.

Poinsot, T. \& Lele, S. 1992 Boundary conditions for direct simulations of compressible viscous flows. J. Comput. Phys. 101 (1), 104-129.

Poinsot, T. \& Veynante, D. 2011 Theoretical and Numerical Combustion. Third Edition (www.cerfacs.fr/elearning).

Polifke, W., Paschereit, C. \& Doebbeling, K. 2001 Constructive and destructive interference of acoustic and entropy waves in a premixed combustor with a choked exit. Int. J. Acoust. Vib. 6, 135-146.

RAYleigh, L. 1878 The explanation of certain acoustic phenomena. Nature July 18, 319-321.

Rowley, C. W., Mezic̀, I., Bagheri, S., Schlatter, P. \& Henningson, D. S. 2009 Spectral analysis of nonlinear flows. J. Fluid Mech. 641, 115-127.

Sattelmayer, T. 2003 Influence of the combustor aerodynamics on combustion instabilities from equivalence ratio fluctuations. J. Eng. Gas Turb. and Power 125, 11-19.

Schmid, Peter J. 2010 Dynamic mode decomposition of numerical and experimental data. J. Fluid Mech. 656, 5-28.

Schmid, P. J., Li, L., Juniper, M. P. \& Pust, O. 2011 Applications of the dynamic mode decomposition. Theoret. Comput. Fluid Dynamics 25 (1-4), 249-259.

Schmitt, P., Poinsot, T., Schuermans, B. \& Geigle, K. P. 2007 Large-eddy simulation and experimental study of heat transfer, nitric oxide emissions and combustion instability in a swirled turbulent high-pressure burner. J. Fluid Mech. 570, 17-46.

Selimefendigil, Fatih \& Polifke, Wolfgang 2011 A nonlinear frequency domain model for limit cycles in thermoacoustic systems with modal coupling. Int. J. Spray Combust. Dynamics 3 (4), 303-330.

Silva, C. F., Nicoud, F., Schuller, T., Durox, D. \& Candel, S. 2013 Combining a helmholtz solver with the flame describing function to assess combustion instability in a premixed swirled combustor. Combustion and Flame 160 (9), 1743 - 1754.

Sisco, J.C., Yu, Y.C., Sankaran, V. \& Anderson, W.E. 2011 Examination of mode shapes in an unstable model combustor. J. Sound Vib. 330 (1), $61-74$.

Smagorinsky, J. 1963 General circulation experiments with the primitive equations: 1. the basic experiment. Mon. Weather Rev. 91, 99-164.

Tsien, H. S. 1952 The transfer functions of rocket nozzles. J. American Rocket Society 22 (3), 139-143.

Vuillot, F. 1995 Vortex-shedding phenomena in solid rocket motors. J. Prop. Power 11 (4), 626-639.

Wolf, P., Staffelbach, G., Gicquel, L. Y. M., Müller, J. D. \& Poinsot, T. 2012 Acoustic and large eddy simulation studies of azimuthal modes in annular combustion chambers. Combust. Flame 159 (11), 3398-3413.

Yao, Z., Gao, Y., Zhu, M., Dowling, A. P. \& Bray, K. N. C. 2012 Combustion rumble prediction with integrated computational-fluid-dynamics/low-order-model methods. J. Prop. Power 28 (5), 1015-1025.

You, D., Huang, Y. \& YAng, V. 2005 A generalized model of acoustic reponse of turbulent premixed flame and its application to gas-turbine combustion instability analysis. Combust. Sci. Tech. 177 (5-6), 1109-1150.

Yu, K. H., Trouvé, A. \& DAILY, J. W. 1991 Low-frequency pressure oscillations in a model ramjet combustor. J. Fluid Mech. 232, 47-72.

Yu, Y. C., Sisco, J. C., Sankaran, V. \& Anderson, W. E. 2010 Effects of mean flow, entropy waves, and boundary conditions on longitudinal combustion instability. Combust. Sci. Tech. $182(7), 739-776$.

Zhu, M., Dowling, A. P. \& Bray, K. N. C. 2001 Self-excited oscillations in combustors with spray atomizers. J. Eng. Gas Turb. and Power 123 (4), 779-786.

Zinn, B.T. 1972 Longitudinal mode acoustic losses in short nozzles. J. Sound Vib. 22 (1), 93 -105 .

Zinn, B. T., Bell, W. A., Daniel, B. R. \& Smith, A. J. 1973 Experimental determination of three-dimensional liquid rocket nozzle admittances. AIAA Journal 11, 267-272. 